\title{
Structured Parallel Programming
}

— Yong Wang -
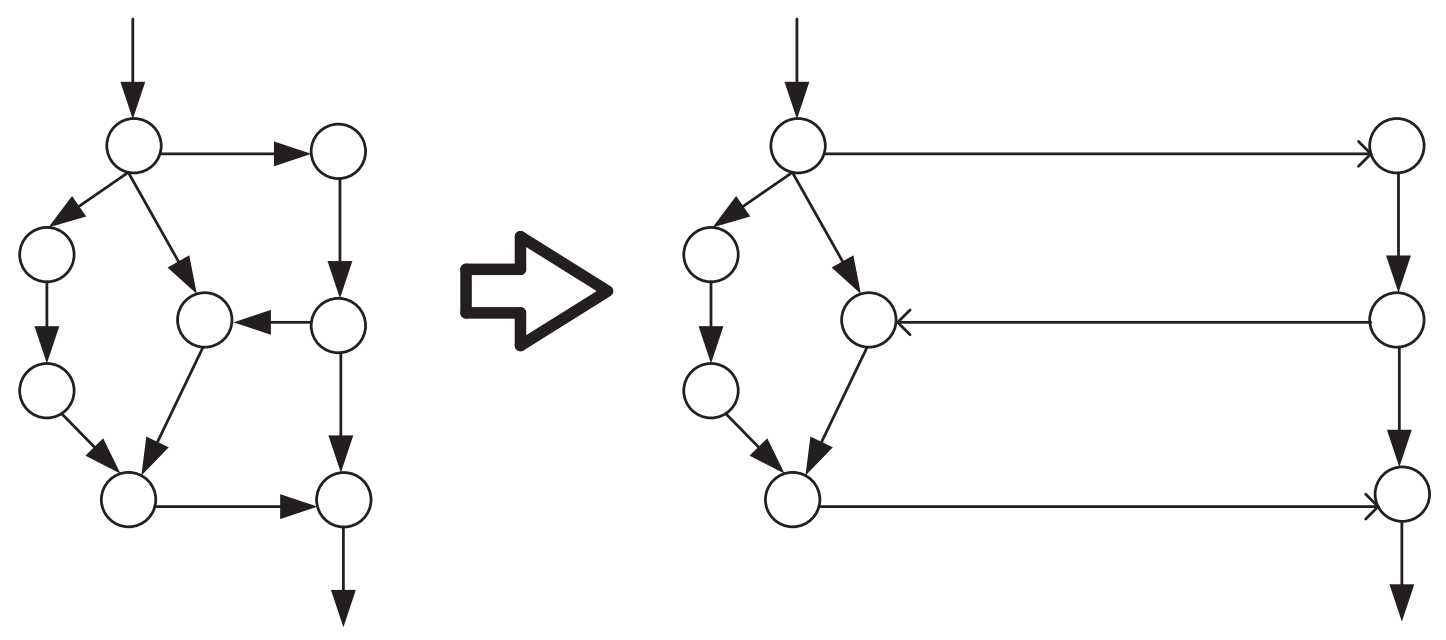


\section{Contents}

1 Introduction 1

2 Parallelism and Concurrency 2

2.1 Unstructured Parallelism - True Concurrency . . . . . . . . . . . . . . . 2 2

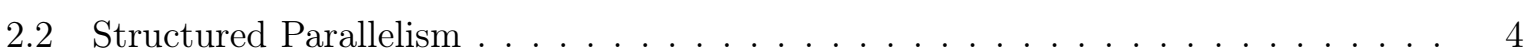

2.3 From Unstructured Parallelism to Structured Parallelism . . . . . . . . . . . . . . 6

2.4 Foundation of Unstructured and Structured Parallelism . . . . . . . . . . . . . . 9

3 A Parallel Programming Language $\quad 10$

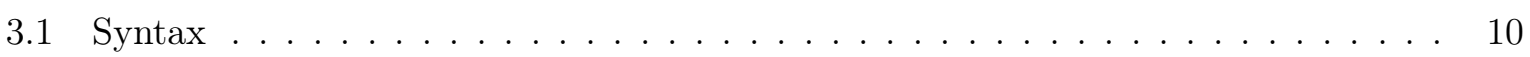

3.2 Operational Semantics . . . . . . . . . . . . . . . . . . . 11

3.2 .1 Operational Rules of $\mathbf{A} \exp \ldots \ldots \ldots \ldots \ldots$

3.2 .2 Operational Rules of $\operatorname{Bexp} \ldots \ldots \ldots \ldots \ldots$

3.2 .3 Operational Rules for Com . . . . . . . . . . . . . . 12

3.3 Denotational Semantics . . . . . . . . . . . . . . . . . . . . . . . . . 16

3.3.1 Denotational Semantics of $\mathbf{A} \exp \ldots \ldots \ldots \ldots \ldots$

3.3.2 Denotational Semantics of Bexp . . . . . . . . . . . . . . 17

3.3.3 Denotational Semantics of Com . . . . . . . . . . . . . . . . . . . 17

3.4 Relations between Operational and Denotational Semantics . . . . . . . . . . . 19

3.5 Axiomatic Semantics . . . . . . . . . . . . . . . . . . . 20

3.5.1 Extended Hoare Rules for Parallelism . . . . . . . . . . . . . . . . . . 20

3.5.2 Soundness of The Extended Hoare Rules . . . . . . . . . . . . . . . . . 21

3.5.3 Completeness of The Extended Hoare Rules . . . . . . . . . . . . . . . . 21

3.6 Non-determinism . . . . . . . . . . . . . . . . . . . . . . . . 22

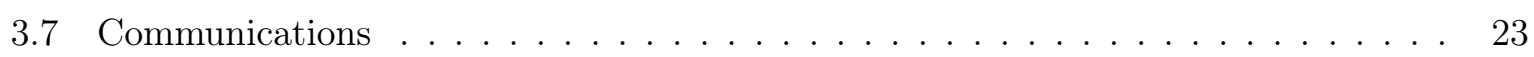

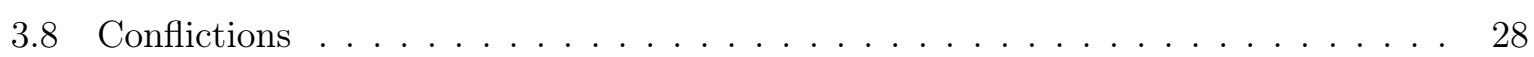

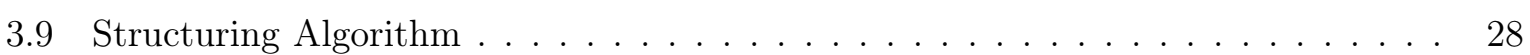




\section{Introduction}

Parallel computing [1 [2] is becoming more and more important. Traditional parallelism often existed in distributed computing, since distributed systems are usually autonomous and local computer is single-core and single-processor and timed (Timed computing is serial in nature). Today, due to the progress of hardware, multi-cores, multi-processors, and GPUs make the local computer truly parallel.

Parallel programming language has a relatively long research history. There have been always two ways: one is the structured way, and the other is the graph-based (true concurrent) way. The structured way is often based on the interleaving semantics, such as process algebra CCS. Since the parallelism in interleaving semantics is not a fundamental computational pattern (the parallel operator can be replaced by alternative composition and sequential composition), the parallel operator often does not occur as an explicit operator, such as the mainstream programming languages $\mathrm{C}, \mathrm{C}++$, Java, et al.

The graph-based way is also called true concurrency [3] [4] [5]. There also have been some ways to structure the graph [6] [7, but these work only considered the causal relation in the graph, and neglected the confliction and even the communication. And there are also industrial efforts to adopt the graph-based way, such as the workflow description language WSFL. The later workflow description language BPEL adopts both the structured way and the graph-based way. Why does BPEL not adopt the structured way only? It is because that the expressive power of the structured way is limited. Then why does BPEL not adopt the graph-based way only? It is just because that the graph could not be structured at that time and the structured way is the basis on implementing a compiler.

We did some work on truly concurrent process algebra [8], which proved that truly concurrent process algebra is a generalization of traditional process algebra and had a side effect on the structuring true concurrency.

Now, it is the time to do some work on structured parallel programming under the background of programming language and parallel software engineering. On one side, traditional structured programming got great successes in sequential computation [9] [10]; on the other side, current structured parallel programming focused on parallel patterns (also known as parallel skeletons, templates, archetypes) [11] [12] [13] [14 [15], with comparison to structured sequential programming, the corresponding structured parallel programming with solid foundation still is missing.

In this paper, we try to clarify structured parallel programming corresponding to traditional structured sequential programming. This paper is organized as follows. In section 2, we introduce the backgrounds of structured and unstructured parallelism. We introduce a parallel programming language called PPL in section 3 . 


\section{Parallelism and Concurrency}

In this section, we analyze the concepts of parallelism and concurrency, unstructured parallelism and structured parallelism.

We introduce unstructured parallelism in section 2.1, structured parallelism in section 2.2, and the way from unstructured parallelism to structured parallelism in section 2.3. In section 2.4. we give the foundation of unstructured and structured parallel computation.

\subsection{Unstructured Parallelism - True Concurrency}

True concurrency is usually defined by a graph-like structure [4] 5], such as DAG (Directed Acyclic Graph), Petri net and event structure. As follows, we give the definition of Prime Event Structure.

Definition 2.1 (Prime event structure). Let $\Lambda$ be a fixed set of labels, ranged over a, .. A ( $\Lambda$ labelled) prime event structure is a tuple $\mathcal{E}=\langle\mathbb{E}, \leq, \sharp, \lambda\rangle$, where $\mathbb{E}$ is a denumerable set of events. Let $\lambda: \mathbb{E} \rightarrow \Lambda$ be a labelling function. And $\leq, \sharp$ are binary relations on $\mathbb{E}$, called causality and conflict respectively, such that:

1. $\leq$ is a partial order and $\lceil e\rceil=\left\{e^{\prime} \in \mathbb{E} \mid e^{\prime} \leq e\right\}$ is finite for all $e \in \mathbb{E}$.

2. $\sharp$ is irreflexive, symmetric and hereditary with respect to $\leq$, that is, for all $e, e^{\prime}, e^{\prime \prime} \in \mathbb{E}$, if $e \sharp e^{\prime} \leq e^{\prime \prime}$, then $e \sharp e^{\prime \prime}$.

Then, the concepts of consistency and concurrency can be drawn from the above definition:

1. $e, e^{\prime} \in \mathbb{E}$ are consistent, denoted as $e \sim e^{\prime}$, if $\neg\left(e \sharp e^{\prime}\right)$. A subset $X \subseteq \mathbb{E}$ is called consistent, if $e-e^{\prime}$ for all $e, e^{\prime} \in X$.

2. $e, e^{\prime} \in \mathbb{E}$ are concurrent, denoted as $e \| e^{\prime}$, if $\neg\left(e \leq e^{\prime}\right), \neg\left(e^{\prime} \leq e\right)$, and $\neg\left(e \sharp e^{\prime}\right)$.

In the Prime Event Structure defined true concurrency, we can see that there exist two kinds of unstructured relations: causality and confliction. Figure 1 and Figure 2 illustrates these two kinds of concurrency (for the simplicity, we separate the causal relation and the conflict relation).

Figure 1 illustrates an example of primitives (atomic actions, events) with causal relations. Note that, primitives, atomic actions and events are almost the same concepts under different backgrounds of computer science, and we will use them with no differences.

Figure 2 illustrates an example of atomic actions with causal relations and conflict relations. There exists a conflict relation between the second action in the left parallel branch and the second action in the right parallel branch, if the condition $b$ is true, then the second action and its subsequent actions in the left branch can execute, else the second action and its subsequent actions in the right branch will execute. 


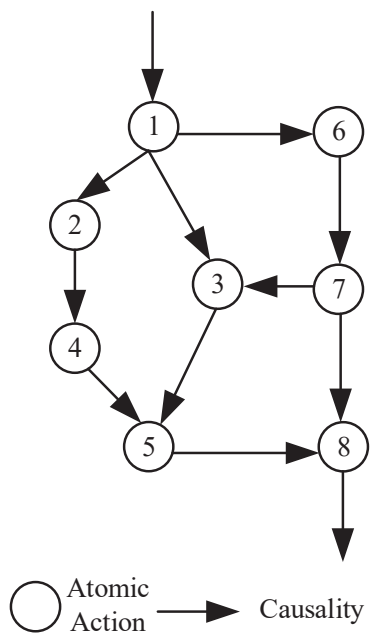

Figure 1: An example of unstructured parallelism

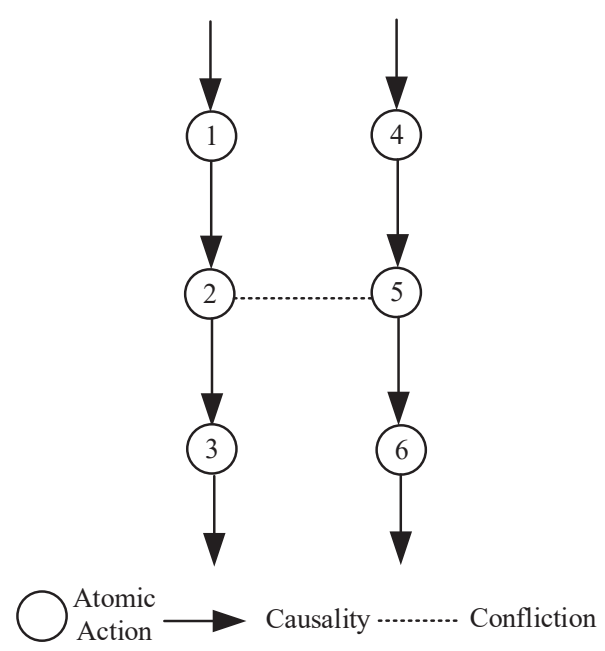

Figure 2: Another example of unstructured parallelism 


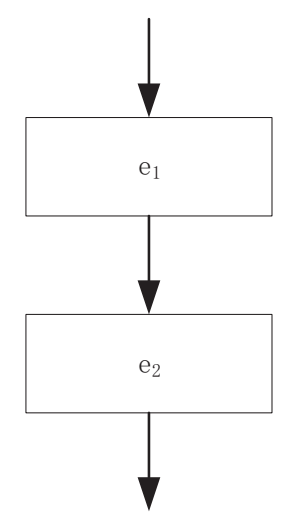

Figure 3: Sequence structure

\subsection{Structured Parallelism}

Comparing to structured programming in sequential computation [9] [10], we can intuitionally add a structured parallelism to the existed three basic programming structures (sequence, choice and iteration) of structured sequential programming, to form four basic programming structures of structured parallel programming: sequence, choice, iteration and parallelism. The intuitions and flow charts of the four basic structures are as follow.

The intuition of sequence (;) of two clauses $e_{1} ; e_{2}$ is that after the successful execution of $e_{1}, e_{2}$ executes. The corresponding flow chat is shown in Figure 3 ,

The intuition of choice if $(b)$ then $e_{1}$ else $e_{2}$ is that if the condition $b$ is true, then $e_{1}$ executes, else $e_{2}$ executes. The corresponding flow chat is shown in Figure 4.

The intuition of iteration while $(b)$ do $e_{1}$ is that while the condition $b$ is true, then $e_{1}$ executes many times. The corresponding flow chat is shown in Figure 5 ,

The intuition of parallelism ( $\|)$ of two clauses $e_{1} \| e_{2}$ is that $e_{1}$ and $e_{2}$ execute simultaneously. The corresponding flow chat is shown in Figure 6.

The programming of atomic actions, mixed by the above four structures is called structured parallel programming. We define Structured Parallel Program inductively as follows.

Definition 2.2 (Structured parallel program). Let the set of all primitives denote $\mathbb{P}$. A Structured Parallel Program SPP is inductively defined as follows:

1. $\mathbb{P} \subset S P P$;

2. If $e_{1} \in S P P$ and $e_{2} \in S P P$, then $e_{1} ; e_{2} \in S P P$;

3. If $b$ is a condition, $e_{1} \in S P P$ and $e_{2} \in S P P$, then if (b) then $e_{1}$ else $e_{2} \in S P P$;

4. If $b$ is a condition, $e \in S P P$, then while $(b)$ do $e \in S P P$;

5. If $e_{1} \in S P P$ and $e_{2} \in S P P$, then $e_{1} \| e_{2} \in S P P$. 


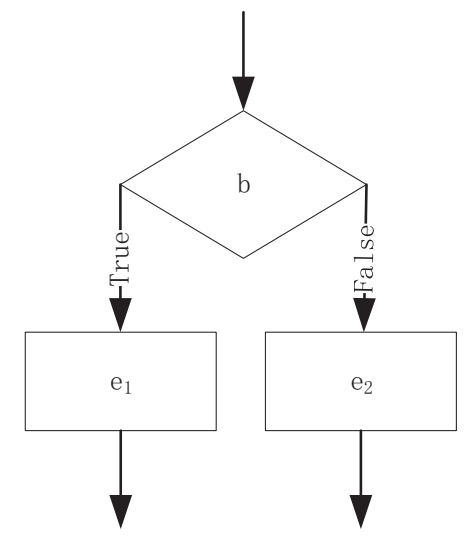

Figure 4: Choice structure

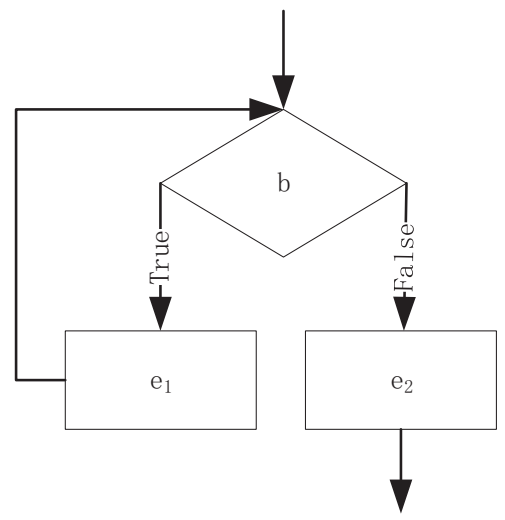

Figure 5: Iteration structure

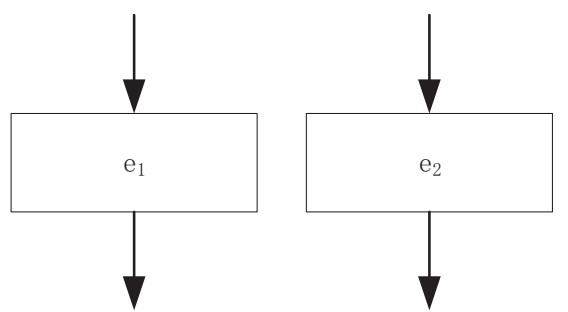

Figure 6: Parallelism structure 


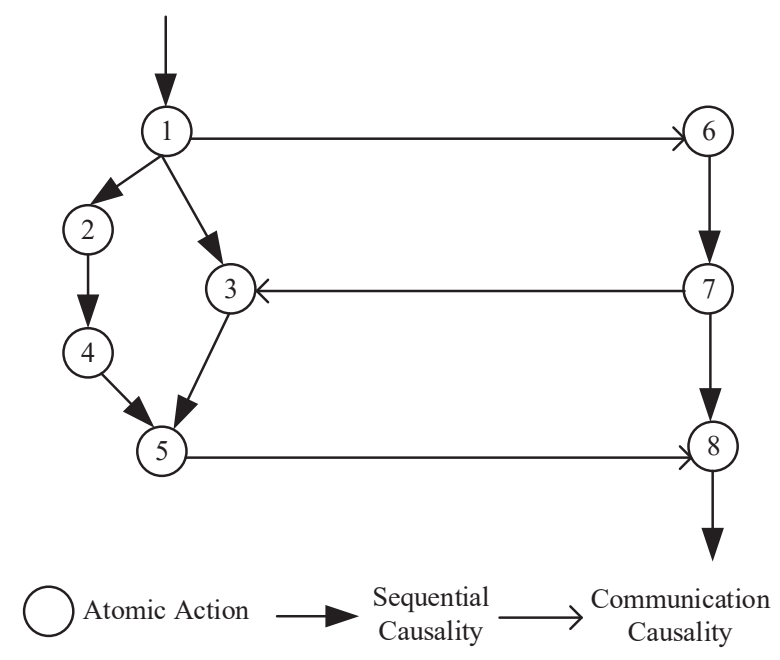

Figure 7: An example of structuring unstructured parallelism

\subsection{From Unstructured Parallelism to Structured Parallelism}

The examples in Figure 1 and 2 are two kinds of typical unstructured parallelism. In this section, we try to structure these unstructured parallelism.

Firstly, the unstructured causalities in the same parallel branch can be structured by the famous conclusion that Goto statement is harmful [9] and also the similarly well-known structured (sequential) programming [10]; and for unstructured causalities, we find the example in Figure 1] can not be structured, and the proof is stated in the following conclusions.

Proposition 2.3. The example in Figure 1 can not be structured.

Proof. The actions 3 and 6 have the same causal pioneer 1, they should be in different parallel branches. But, the action 6 is the causal pioneer of the action 3 through the action 7 , so, they should be in the same parallel branch. These cause contradictions.

How can we deal this situation? Yes, we can classify the causal relations into two kinds: one is traditional sequential causality, and the other is the communication between different parallel branches, since the causality between parallel branches being communication is reasonable. Figure 7 is the causality-classified one originated from Figure 1, This classification should be clarified during modelling time, that is, the programmer should draw Figure 7 directly, instead of drawing Figure 1 and then transforming it to Figure 7 , in the modelling phase. Note that, multi-parties communications can be steadied by a series of two-parties communications without any loss.

Then the causality-classified parallelism can be structured, we show the structuring way of synchronous and asynchronous communications.

For synchronous communication, The program corresponding to Figure 1 can be written as follows: 


$$
(1 ;((2 ; 4) \| 3) ; 5) \|(6 ; 7 ; 8)
$$

with three unstructured communications $s c_{1,6}, s c_{7,3}$ and $s c_{5,8}$.

The above program can be structured and equivalent to the following program:

$$
s c_{1,6} ;\left((2 ; 4) \| s c_{7,3}\right) ; s c_{5,8}
$$

We can see that the above program is structured, though the equivalence of the above two programs is not obvious. We will explain it through an rigorous way in the following chapters.

For asynchronous communication, the program corresponding to Figure 1 can be written as follows:

$$
(1 ;((2 ; 4) \| 3) ; 5) \|(6 ; 7 ; 8)
$$

with three unstructured constraints $1 \leq 6,7 \leq 3$ and $5 \leq 8$. Note that, $\leq$ is the causal relation.

The above program can be structured and equivalent to the following program:

$(1 ;((2 ; 4) \|$ if $(7 \leq 3)$ then 3 else skip $) ; 5) \|($ if $(1 \leq 6)$ then 6 else skip; 7 ; if $(5 \leq 8)$ then 8 else skip).

Note that skip is a voidness primitive.

The above conditions, like $1 \leq 6,7 \leq 3$ and $5 \leq 8$, are not based on the the traditional results of data manipulation. Asynchronous communications are usually implemented by inserting an intermediate data structure, like mailbox or queue, between the two communicating partners, so, the above conditions can be the results of checking the data structure if the data are received in the data structure by the receiver. If the receiver has the ability to be blocked until the data are received, then the above conditions can be removed, and the structured program is the original one:

$$
(1 ;((2 ; 4) \| 3) ; 5) \|(6 ; 7 ; 8)
$$

without any constraint.

Then, it is turn to consider the unstructured conflictions between different parallel branches, since it is already proven that conflictions in the same parallel branch can be structured [10], as the choice structure is a kind of structured confliction. Figure 2 illustrates this kind of unstructured conflictions and can be expressed by the following program:

$$
(1 ; 2 ; 3) \|(4 ; 5 ; 6)
$$

with an unstructured confliction $2 \sharp 5$, and a condition $b$, if $b$ is true then the primitive 2 and its successors execute, else the primitive 5 and its successors execute.

Figure 2 can be structured by Figure 8 . The structured program corresponding to Figure 8 is: if (b) then $(1 ; 2 ; 3) \| 4$ else $1 \|(4 ; 5 ; 6)$ 


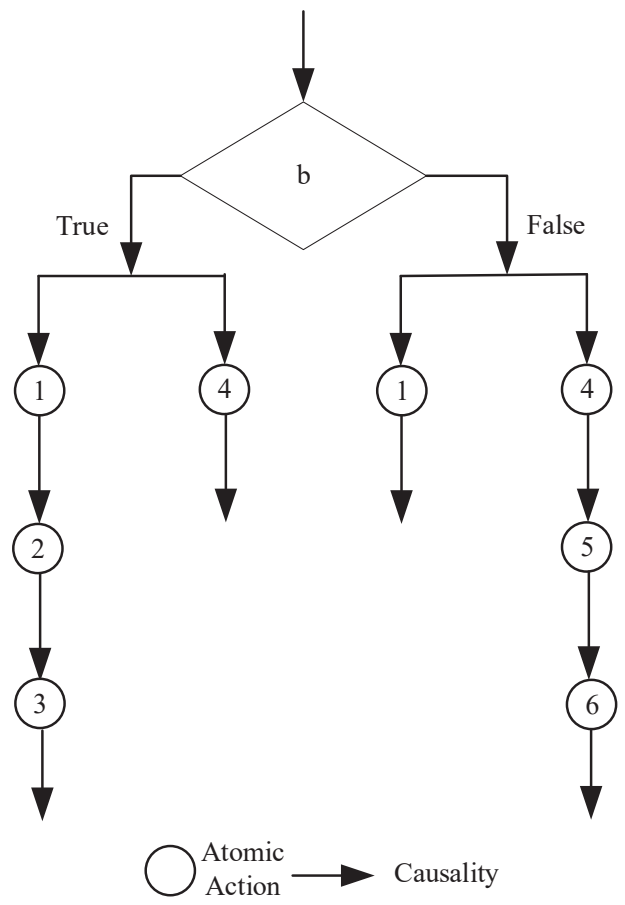

Figure 8: Another example of structuring unstructured parallelism 


\subsection{Foundation of Unstructured and Structured Parallelism}

There existed several parallel machines [16] [17] to provide the foundation for unstructured and structured parallelism since quite long time ago. Among them, the one (or multi)-tapes multiheads Turing machine called PTM (Parallel Turing Machine) [16] provides an intuitive foundation. The unstructured causalities and conflicts can be modelled as communications among the tape heads.

Prather [18] builded the so-called structured Turing machines with the four basic structures (sequence, choice, iteration and parallelism), which can realize every partial recursive function by a structured connection of simple machines. 


\section{A Parallel Programming Language}

In this section, we design a detailed parallel programming language, abbreviated PPL. PPL includes the four basic structures: sequence, choice, iteration and parallelism, and also nondeterminism, communications (causalities between different parallel branches) and conflictions between different parallel branches. Note that, for the integrity, the semantics of traditional parts are also involved.

In section 3.1, we give the syntax of PPL. We give the operational semantics and denotational semantics in section 3.2 and 3.3, and the relation between them in section 3.4, we give the axiomatic semantics in section 3.5. We discuss non-determinism in section 3.6, communications in section 3.7 and conflictions in section 3.8, and the structuring algorithm in section 3.9.

\subsection{Syntax}

The syntactic sets of PPL are as follows.

- Numbers set $\mathbf{N}$, with positive, negative integers and zero, and $n, m \in \mathbf{N}$;

- Truth values set $\mathbf{T}$, with values $\{$ true, false $\}$;

- Storage locations Loc, and $X, Y \in \mathbf{L o c}$;

- Arithmetic expressions Aexp, and $a \in$ Aexp;

- Boolean expressions Bexp, and $b \in \mathbf{B e x p}$;

- Commands Com, and $c \in \mathbf{C o m}$.

The formation rules of PPL are:

For Aexp:

$$
a::=n \quad|\quad X \quad| \quad a_{0}+a_{1} \mid \begin{array}{lll}
a_{0}-a_{1} & \mid a_{0} \times a_{1}
\end{array}
$$

For Bexp:

$$
b::=\text { true } \quad \text { false }\left|a_{0}=a_{1} \quad\right| \quad a_{0} \leq a_{1} \quad|\quad \neg b \quad| \quad b_{0} \wedge b_{1} \quad \mid \quad b_{0} \vee b_{1}
$$

For Com:

$$
c::=\text { skip } \quad|\quad X:=a \quad| \quad c_{0} ; c_{1} \quad \mid \quad \text { if } b \text { then } c_{0} \text { else } c_{1} \mid \text { while } b \text { do } c \quad \mid \quad c_{0} \| c_{1}
$$

We see that the syntax of PPL is almost same to traditional imperative language, except for the explicit parallel operator $\|$ in Com. 


\subsection{Operational Semantics}

The set of states $\Sigma$ are composed of $\sigma: \mathbf{L o c} \rightarrow \mathbf{N}$, so, $\sigma(X)$ is the values of storage location $X$ under the state $\sigma$. For more about operational semantics, please refer to Plotkin's book [19].

In this section, we give the operational semantics of PPL.

\subsubsection{Operational Rules of Aexp}

$\langle a, \sigma\rangle$ is called the configuration of arithmetic expression $a$, while $\langle a, \sigma\rangle \rightarrow n$ denotes that the value of $a$ is $n$ under the state $\sigma$.

The evaluation rule of integer $n$ :

$$
\langle n, \sigma\rangle \rightarrow n
$$

The evaluation rule of storage location $X$ :

$$
\langle X, \sigma\rangle \rightarrow \sigma(X)
$$

The evaluation rule of sums:

$$
\frac{\left\langle a_{0}, \sigma\right\rangle \rightarrow n_{0} \quad\left\langle a_{1}, \sigma\right\rangle \rightarrow n_{1}}{\left\langle a_{0}+a_{1}, \sigma\right\rangle \rightarrow n}, n=n_{0}+n_{1}
$$

The evaluation rule of subtractions:

$$
\frac{\left\langle a_{0}, \sigma\right\rangle \rightarrow n_{0} \quad\left\langle a_{1}, \sigma\right\rangle \rightarrow n_{1}}{\left\langle a_{0}-a_{1}, \sigma\right\rangle \rightarrow n}, n=n_{0}-n_{1}
$$

The evaluation rule of products:

$$
\frac{\left\langle a_{0}, \sigma\right\rangle \rightarrow n_{0} \quad\left\langle a_{1}, \sigma\right\rangle \rightarrow n_{1}}{\left\langle a_{0} \times a_{1}, \sigma\right\rangle \rightarrow n}, n=n_{0} \times n_{1}
$$

Then we can define the following equivalence $\sim$ as follows.

Definition 3.1 (Equivalence of operational semantics for arithmetic expressions). $a_{0} \sim a_{1}$ iff $\forall n \in \mathbf{N}, \forall \sigma \in \Sigma .\left\langle a_{0}, \sigma\right\rangle \rightarrow n \Leftrightarrow\left\langle a_{1}, \sigma\right\rangle \rightarrow n$.

\subsubsection{Operational Rules of Bexp}

The evaluation rule of true:

$$
\langle\text { true, } \sigma\rangle \rightarrow \text { true }
$$

The evaluation rule of false:

$$
\langle\text { false, } \sigma\rangle \rightarrow \text { false }
$$


The evaluation rule of equality:

$$
\begin{gathered}
\frac{\left\langle a_{0}, \sigma\right\rangle \rightarrow n_{0} \quad\left\langle a_{1}, \sigma\right\rangle \rightarrow n_{1}}{\left\langle a_{0}=a_{1}, \sigma\right\rangle \rightarrow \text { true }}, n_{0}=n_{1} \\
\frac{\left\langle a_{0}, \sigma\right\rangle \rightarrow n_{0} \quad\left\langle a_{1}, \sigma\right\rangle \rightarrow n_{1}}{\left\langle a_{0}=a_{1}, \sigma\right\rangle \rightarrow \text { false }}, n_{0} \neq n_{1}
\end{gathered}
$$

The evaluation rule of $\leq$ :

$$
\begin{gathered}
\frac{\left\langle a_{0}, \sigma\right\rangle \rightarrow n_{0} \quad\left\langle a_{1}, \sigma\right\rangle \rightarrow n_{1}}{\left\langle a_{0} \leq a_{1}, \sigma\right\rangle \rightarrow \text { true }}, n_{0} \leq n_{1} \\
\frac{\left\langle a_{0}, \sigma\right\rangle \rightarrow n_{0} \quad\left\langle a_{1}, \sigma\right\rangle \rightarrow n_{1}}{\left\langle a_{0} \leq a_{1}, \sigma\right\rangle \rightarrow \text { false }}, n_{0} \geq n_{1}
\end{gathered}
$$

The evaluation rule of $\neg$ :

$$
\begin{gathered}
\frac{\langle b, \sigma\rangle \rightarrow \text { true }}{\langle\neg b, \sigma\rangle \rightarrow \text { false }} \\
\frac{\langle b, \sigma\rangle \rightarrow \text { false }}{\langle\neg b, \sigma\rangle \rightarrow \text { true }}
\end{gathered}
$$

The evaluation rule of $\wedge$ :

$$
\frac{\left\langle b_{0}, \sigma\right\rangle \rightarrow t_{0} \quad\left\langle b_{1}, \sigma\right\rangle \rightarrow t_{1}}{\left\langle b_{0} \wedge b_{1}, \sigma\right\rangle \rightarrow t}, t=\text { true }, t_{0} \equiv \text { true } \wedge t_{1} \equiv \text { true } ; t=\text { false, } \text { otherwise }
$$

The evaluation rule of $\mathrm{v}$ :

$$
\frac{\left\langle b_{0}, \sigma\right\rangle \rightarrow t_{0} \quad\left\langle b_{1}, \sigma\right\rangle \rightarrow t_{1}}{\left\langle b_{0} \vee b_{1}, \sigma\right\rangle \rightarrow t}, t=\text { true, } t_{0} \equiv \text { true } \vee t_{1} \equiv \text { true } ; t=\text { false, } \text { otherwise }
$$

Then we can define the following equivalence $~$ as follows.

Definition 3.2 (Equivalence of operational semantics for boolean expressions). $b_{0} \sim b_{1}$ iff $\forall t \epsilon$ $\mathbf{T}, \forall \sigma \in \Sigma .\left\langle b_{0}, \sigma\right\rangle \rightarrow t \Leftrightarrow\left\langle b_{1}, \sigma\right\rangle \rightarrow t$.

\subsubsection{Operational Rules for Com}

$\langle c, \sigma\rangle$ denotes the configuration of the command $c$, which means that the command $c$ executes under the state $\sigma$. And $\langle c \sigma\rangle \rightarrow \sigma^{\prime}$ means that the command $c$ executing under the state $\sigma$ evolves to the state $\sigma^{\prime}$. For $n \in \mathbf{N}$ and $X \in \mathbf{L o c}, \sigma[n / X]$ denotes using $n$ to replace the contents of $X$ under the state $\sigma$.

The execution rule of skip:

$$
\langle\text { skip, } \sigma\rangle \rightarrow \sigma
$$


The execution rule of assignment:

$$
\frac{\langle a, \sigma\rangle \rightarrow n}{\langle X:=a, \sigma\rangle \rightarrow \sigma[n / X]}
$$

The execution rule of sequence:

$$
\frac{\left\langle c_{0}, \sigma\right\rangle \rightarrow \sigma^{\prime}}{\left\langle c_{0} ; c_{1}, \sigma\right\rangle \rightarrow\left\langle c_{1}, \sigma^{\prime}\right\rangle}
$$

The execution rule of choice:

$$
\begin{gathered}
\frac{\langle b, \sigma\rangle \rightarrow \text { true } \quad\left\langle c_{0}, \sigma\right\rangle \rightarrow \sigma^{\prime}}{\left\langle\text { if } b \text { then } c_{0} \text { else } c_{1}, \sigma\right\rangle \rightarrow \sigma^{\prime}} \\
\frac{\langle b, \sigma\rangle \rightarrow \text { false } \quad\left\langle c_{1}, \sigma\right\rangle \rightarrow \sigma^{\prime}}{\left\langle\text { if } b \text { then } c_{0} \text { else } c_{1}, \sigma\right\rangle \rightarrow \sigma^{\prime}}
\end{gathered}
$$

The execution rule of iteration:

$$
\begin{gathered}
\frac{\langle b, \sigma\rangle \rightarrow \text { false }}{\langle\text { while } b \text { do } c, \sigma\rangle \rightarrow \sigma} \\
\frac{\langle b, \sigma\rangle \rightarrow \text { true } \quad\langle c, \sigma\rangle \rightarrow \sigma^{\prime \prime} \quad\left\langle\text { while } b \text { do } c, \sigma^{\prime \prime}\right\rangle \rightarrow \sigma^{\prime}}{\langle\text { while } b \text { do } c, \sigma\rangle \rightarrow \sigma^{\prime}}
\end{gathered}
$$

The execution rule of parallelism:

$$
\frac{\left\langle c_{1}, \sigma\right\rangle \rightarrow \sigma^{\prime} \quad\left\langle c_{0}, \sigma\right\rangle \rightarrow \sigma^{\prime \prime}}{\left\langle c_{0} \| c_{1}, \sigma\right\rangle \rightarrow \sigma^{\prime} \uplus \sigma^{\prime \prime}}
$$

where $\sigma^{\prime} \uplus \sigma^{\prime \prime}$ is the final states after $c_{0}$ and $c_{1}$ execute simultaneously.

Note that, for true concurrency, there are still three other properties should be processed: communications, conflictions, and race conditions (we leave them to the next section).

1. Communication is occurring between two atomic communicating commands, which can be defined by a communication function $\gamma\left(c_{0}, c_{1}\right) \triangleq c\left(c_{0}, c_{1}\right)$. Communications can be implemented by several ways: share storage locations, invocation of functions by values, or network communications. For a pure imperative programming lange, we only consider the case of share storage locations, so, there is no need to define new communicating commands. So, two commands in communication is with a relation $\gamma\left(c_{0}, c_{1}\right) \triangleq c\left(c_{0}, c_{1}\right)$, but rules of $c_{0} \| c_{1}$ are still the same to the above ones. We will discuss the general communications in section 3.7.

2. Confliction may have two forms: one exists as the condition rules define; the other may exist among the parallel branches, which must be eliminated. But the elimination of confliction existing in parallel branches may lead to non-deterministic results (refer to [8] for details). For simplicity, we assume that the programs written by PPL at this time have 
no conflictions, because a program with the conflictions existing among parallel branches have an equal program without conflicts. That is, the conflictions can be eliminated and structured, and we will discuss the elimination of conflictions between parallel branches in section 3.8

3. Race condition may exist in two parallel commands, for example, they are all executing assignment to a same storage location. Two parallel commands in race condition must be executed serially. We should define new rules for race condition, but, these rules also lead to non-deterministic results. So, we also assume that the programs written by PPL deal with this situation and the non-deterministic execution is eliminated. In fact, we can write $c_{0} \|\left(\mathbf{s k i p} ; c_{1}\right)$ or $\left(\mathbf{s k i p} ; c_{0}\right) \| c_{1}$, or put $c_{0}, c_{1}$ in a condition, where $c_{0}$ and $c_{1}$ are in race condition. But, indeed, the above parallelism is still can be used widely in nonsharing memory computation (distributed computing), or non-racing of sharing memory computation. For the general form of non-determinism, we will discuss in section [3.6 and 3.7.

We can get the following propositions. Where $\sim$ is an equivalence relation on commands by the definition, where $\Sigma$ is the set of states:

Definition 3.3 (Equivalence of operational semantics for commands). $c_{0} \sim c_{1}$ iff $\forall \sigma, \sigma^{\prime} \in \Sigma,\left\langle c_{0}, \sigma\right\rangle \rightarrow$ $\sigma^{\prime} \Leftrightarrow\left\langle c_{1}, \sigma\right\rangle \rightarrow \sigma^{\prime}$

Proposition 3.4. $c_{0}\left\|c_{1} \sim c_{1}\right\| c_{0}$, for $c_{0}, c_{1} \in \mathbf{C o m}$.

Proof. By use of the transition rules of $\|$, we can get the following derivations of $c_{0} \| c_{1}$ for $\forall \sigma \in \Sigma:$

$$
\frac{\left\langle c_{1}, \sigma\right\rangle \rightarrow \sigma^{\prime} \quad\left\langle c_{0}, \sigma\right\rangle \rightarrow \sigma^{\prime \prime}}{\left\langle c_{0} \| c_{1}, \sigma\right\rangle \rightarrow \sigma^{\prime} \uplus \sigma^{\prime \prime}}
$$

And we can get the following derivations of $c_{1} \| c_{0}$ for $\forall \sigma \in \Sigma$ :

$$
\frac{\left\langle c_{0}, \sigma\right\rangle \rightarrow \sigma^{\prime} \quad\left\langle c_{1}, \sigma\right\rangle \rightarrow \sigma^{\prime \prime}}{\left\langle c_{1} \| c_{0}, \sigma\right\rangle \rightarrow \sigma^{\prime} \uplus \sigma^{\prime \prime}}
$$

So, it is obvious that $c_{0}\left\|c_{1} \sim c_{1}\right\| c_{0}$, for $c_{0}, c_{1} \in \mathbf{C o m}$, as desired.

Proposition 3.5. $\left(c_{0} \| c_{1}\right)\left\|c_{2} \sim c_{0}\right\|\left(c_{1} \| c_{2}\right)$, for $c_{0}, c_{1}, c_{2} \in \mathbf{C o m}$.

Proof. By use of the transition rules of $\|$, we can get the following derivations of $\left(c_{0} \| c_{1}\right) \| c_{2}$ for $\forall \sigma \in \Sigma$ :

$$
\frac{\left\langle c_{0}, \sigma\right\rangle \rightarrow \sigma^{\prime} \quad\left\langle c_{1}, \sigma\right\rangle \rightarrow \sigma^{\prime \prime} \quad\left\langle c_{2}, \sigma\right\rangle \rightarrow \sigma^{\prime \prime \prime}}{\left\langle\left(c_{0} \| c_{1}\right) \| c_{2}, \sigma\right\rangle \rightarrow \sigma^{\prime} \uplus \sigma^{\prime \prime} \uplus \sigma^{\prime \prime \prime}}
$$

And we can get the following derivations of $c_{0} \|\left(c_{1} \| c_{2}\right)$ for $\forall \sigma \in \Sigma$ :

$$
\frac{\left\langle c_{0}, \sigma\right\rangle \rightarrow \sigma^{\prime} \quad\left\langle c_{1}, \sigma\right\rangle \rightarrow \sigma^{\prime \prime} \quad\left\langle c_{2}, \sigma\right\rangle \rightarrow \sigma^{\prime \prime \prime}}{\left\langle c_{0} \|\left(c_{1} \| c_{2}\right), \sigma\right\rangle \rightarrow \sigma^{\prime} \uplus \sigma^{\prime \prime} \uplus \sigma^{\prime \prime \prime}}
$$

So, it is obvious that $\left(c_{0} \| c_{1}\right)\left\|c_{2} \sim c_{0}\right\|\left(c_{1} \| c_{2}\right)$, for $c_{0}, c_{1}, c_{2} \in \mathbf{C o m}$, as desired. 
Proposition 3.6. (if $b$ then $c_{0}$ else $c_{1}$ ) $\| c_{2} \sim$ if $b$ then $c_{0} \| c_{2}$ else $c_{1} \| c_{2}$, for $c_{0}, c_{1}, c_{2} \epsilon$ Com

Proof. By use of the transition rules of choice and $\|$, we can get the following derivations of (if $b$ then $c_{0}$ else $c_{1}$ ) $\| c_{2}$ :

$$
\begin{aligned}
& \frac{\langle b, \sigma\rangle \rightarrow \text { true } \quad\left\langle c_{0}, \sigma\right\rangle \rightarrow \sigma^{\prime} \quad\left\langle c_{2}, \sigma\right\rangle \rightarrow \sigma^{\prime \prime}}{\left\langle\left(\text { if } b \text { then } c_{0} \text { else } c_{1}\right) \| c_{2}, \sigma\right\rangle \rightarrow \sigma^{\prime} \uplus \sigma^{\prime \prime}} \\
& \frac{\langle b, \sigma\rangle \rightarrow \text { false } \quad\left\langle c_{1}, \sigma\right\rangle \rightarrow \sigma^{\prime} \quad\left\langle c_{2}, \sigma\right\rangle \rightarrow \sigma^{\prime \prime}}{\left\langle\left(\text { if } b \text { then } c_{0} \text { else } c_{1}\right) \| c_{2}, \sigma\right\rangle \rightarrow \sigma^{\prime} \uplus \sigma^{\prime \prime}}
\end{aligned}
$$

And we can get the following derivations of if $b$ then $c_{0} \| c_{2}$ else $c_{1} \| c_{2}$ :

$$
\begin{aligned}
& \frac{\langle b, \sigma\rangle \rightarrow \text { true }\left\langle c_{0}, \sigma\right\rangle \rightarrow \sigma^{\prime} \quad\left\langle c_{2}, \sigma\right\rangle \rightarrow \sigma^{\prime \prime}}{\left\langle\left(\text { if } b \text { then } c_{0} \| c_{2} \text { else } c_{1} \| c_{2}, \sigma\right\rangle \rightarrow \sigma^{\prime} \uplus \sigma^{\prime \prime}\right.} \\
& \frac{\langle b, \sigma\rangle \rightarrow \text { false } \quad\left\langle c_{1}, \sigma\right\rangle \rightarrow \sigma^{\prime} \quad\left\langle c_{2}, \sigma\right\rangle \rightarrow \sigma^{\prime \prime}}{\left\langle\left(\text { if } b \text { then } c_{0} \| c_{2} \text { else } c_{1} \| c_{2}, \sigma\right\rangle \rightarrow \sigma^{\prime} \uplus \sigma^{\prime \prime}\right.}
\end{aligned}
$$

So, it is obvious that (if $b$ then $c_{0}$ else $c_{1}$ ) $\| c_{2} \sim$ if $b$ then $c_{0} \| c_{2}$ else $c_{1} \| c_{2}$, for $c_{0}, c_{1}, c_{2} \epsilon$ Com, as desired.

Proposition 3.7. For $c_{0}, c_{1}, c_{2}, c_{3} \in \mathbf{C o m}$,

1. $\left(c_{0} ; c_{1}\right) \| c_{2} \sim\left(c_{0} \| c_{2}\right) ; c_{1}$;

2. $\left(c_{0} ; c_{1}\right) \|\left(c_{2} ; c_{3}\right) \sim\left(c_{0} \| c_{2}\right) ;\left(c_{1} \| c_{3}\right)$.

Proof. (1) By use of the transition rules of sequence and $\|$, we can get the following derivations of $\left(c_{0} ; c_{1}\right) \| c_{2}$ :

$$
\frac{\left\langle c_{0}, \sigma\right\rangle \rightarrow \sigma^{\prime} \quad\left\langle c_{2}, \sigma\right\rangle \rightarrow \sigma^{\prime \prime}}{\left\langle\left(c_{0} ; c_{1}\right) \| c_{2}, \sigma\right\rangle \rightarrow\left\langle c_{1}, \sigma^{\prime} \uplus \sigma^{\prime \prime}\right\rangle}
$$

And we can get the following derivations of $\left(c_{0} \| c_{2}\right) ; c_{1}$ :

$$
\frac{\left\langle c_{0}, \sigma\right\rangle \rightarrow \sigma^{\prime} \quad\left\langle c_{2}, \sigma\right\rangle \rightarrow \sigma^{\prime \prime}}{\left\langle\left(c_{0} \| c_{2}\right) ; c_{1}, \sigma\right\rangle \rightarrow\left\langle c_{1}, \sigma^{\prime} \uplus \sigma^{\prime \prime}\right\rangle}
$$

So, it is obvious that $\left(c_{0} ; c_{1}\right) \| c_{2} \sim\left(c_{0} \| c_{2}\right) ; c_{1}$, for $c_{0}, c_{1}, c_{2} \in \mathbf{C o m}$, as desired.

(2) By use of the transition rules of sequence and $\|$, we can get the following derivations of $\left(c_{0} ; c_{1}\right) \|\left(c_{2} ; c_{3}\right)$ :

$$
\frac{\left\langle c_{0}, \sigma\right\rangle \rightarrow \sigma^{\prime} \quad\left\langle c_{2}, \sigma\right\rangle \rightarrow \sigma^{\prime \prime}}{\left\langle\left(c_{0} ; c_{1}\right) \|\left(c_{2} ; c_{3}\right), \sigma\right\rangle \rightarrow\left\langle c_{1} \| c_{3}, \sigma^{\prime} \uplus \sigma^{\prime \prime}\right\rangle}
$$

And we can get the following derivations of $\left(c_{0} \| c_{2}\right) ;\left(c_{1} \| c_{3}\right)$ : 


$$
\frac{\left\langle c_{0}, \sigma\right\rangle \rightarrow \sigma^{\prime} \quad\left\langle c_{2}, \sigma\right\rangle \rightarrow \sigma^{\prime \prime}}{\left\langle\left(c_{0} \| c_{2}\right) ;\left(c_{1} \| c_{3}\right), \sigma\right\rangle \rightarrow\left\langle c_{1} \| c_{3}, \sigma^{\prime} \uplus \sigma^{\prime \prime}\right\rangle}
$$

So, it is obvious that $\left(c_{0} ; c_{1}\right) \|\left(c_{2} ; c_{3}\right) \sim\left(c_{0} \| c_{2}\right) ;\left(c_{1} \| c_{3}\right)$, for $c_{0}, c_{1}, c_{2}, c_{3} \in \mathbf{C o m}$, as desired.

Proposition 3.8. $c \|$ skip $\sim c$, for $c \in$ Com.

Proof. By use of the transition rules of skip and $\|$, we can get the following derivations of $c \|$ skip:

$$
\frac{\langle c, \sigma\rangle \rightarrow \sigma^{\prime} \quad\langle\text { skip, } \sigma\rangle \rightarrow \sigma}{c \| \text { skip, } \sigma\rangle \rightarrow \sigma^{\prime} \uplus \sigma}
$$

And it is obvious that:

$$
\frac{\langle c, \sigma\rangle \rightarrow \sigma^{\prime}}{c, \sigma\rangle \rightarrow \sigma^{\prime}}
$$

For $\sigma^{\prime} \uplus \sigma=\sigma^{\prime}$, it is obvious that $c \|$ skip $\sim c$, for $c \in \mathbf{C o m}$, as desired.

Lemma 3.9. For $c_{0}, c_{1} \in$ Com,

1. $c_{0}\left\|c_{1} \sim c_{0}\right\|\left(\right.$ skip; $\left.c_{1}\right) \sim c_{0} ; c_{1}$

2. $c_{0}\left\|c_{1} \sim\left(\mathbf{s k i p} ; c_{0}\right)\right\| c_{1} \sim c_{1} ; c_{0}$.

Proof. It is obvious by Proposition 3.7 and 3.8 .

From Lemma 3.15, we can see that the execution orders of $c_{0} \| c_{1}$ cause non-determinism, they can be executed in any sequential order or in parallel simultaneously. But, without race condition, the final states after the execution of $c_{0} \| c_{1}$ are deterministic.

\subsection{Denotational Semantics}

Denotational semantics can be used to describe the semantics of PPL. For more about denotational semantics, please refer to Mosses's book [20].

In this section, we give the denotational semantics for PPL.

\subsubsection{Denotational Semantics of Aexp}

We define the denotational semantics of $\mathbf{A} \exp$ as $\mathcal{A}: \operatorname{Aexp} \rightarrow(\Sigma \rightarrow \mathbf{N})$. The concrete denotational semantics of Aexp are following.

$$
\begin{aligned}
& \mathcal{A}[[n]]=\{(\sigma, n) \mid \sigma \in \Sigma\} \\
& \mathcal{A}[[X]]=\{(\sigma, \sigma(X)) \mid \sigma \in \Sigma\} \\
& \mathcal{A}\left[\left[a_{0}+a_{1}\right]\right]=\left\{\left(\sigma, n_{0}+n_{1}\right) \mid\left(\sigma, n_{0}\right) \in \mathcal{A}\left[\left[a_{0}\right]\right] \&\left(\sigma, n_{1}\right) \in \mathcal{A}\left[\left[a_{1}\right]\right]\right\} \\
& \mathcal{A}\left[\left[a_{0}-a_{1}\right]\right]=\left\{\left(\sigma, n_{0}-n_{1}\right) \mid\left(\sigma, n_{0}\right) \in \mathcal{A}\left[\left[a_{0}\right]\right] \&\left(\sigma, n_{1}\right) \in \mathcal{A}\left[\left[a_{1}\right]\right]\right\} \\
& \mathcal{A}\left[\left[a_{0} \times a_{1}\right]\right]=\left\{\left(\sigma, n_{0} \times n_{1}\right) \mid\left(\sigma, n_{0}\right) \in \mathcal{A}\left[\left[a_{0}\right]\right] \&\left(\sigma, n_{1}\right) \in \mathcal{A}\left[\left[a_{1}\right]\right]\right\}
\end{aligned}
$$




\subsubsection{Denotational Semantics of Bexp}

We define the denotational semantics of $\mathbf{B e x p}$ as $\mathcal{B}: \mathbf{B} \exp \rightarrow(\Sigma \rightarrow \mathbf{T})$. The concrete denotational semantics of Bexp are following.

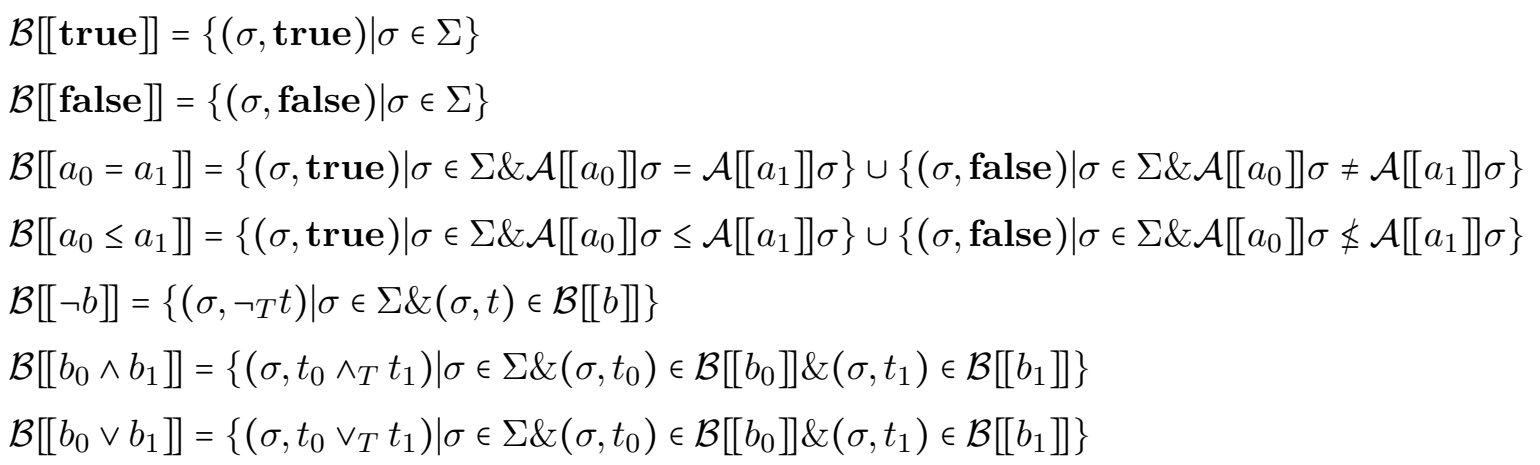

\subsubsection{Denotational Semantics of Com}

We define the denotational semantics of $\mathbf{C o m}$ as $\mathcal{C}: \mathbf{C o m} \rightarrow(\Sigma \rightarrow \Sigma)$. The denotational semantics of Com are following.

$\mathcal{C}[[$ skip $]]=\{(\sigma, \sigma) \mid \sigma \in \Sigma\}$

$\mathcal{C}[[X:=a]]=\{(\sigma, \sigma[n / X]) \mid \sigma \in \Sigma \& n=\mathcal{A}[[a]] \sigma\}$

$\mathcal{C}\left[\left[c_{0} ; c_{1}\right]\right]=\mathcal{C}\left[\left[c_{1}\right]\right] \circ \mathcal{C}\left[\left[c_{0}\right]\right]$

$\mathcal{C}\left[\right.$ if $b$ then $c_{0}$ elsec $\left.\left.c_{1}\right]\right]=\left\{\left(\sigma, \sigma^{\prime}\right) \mid \mathcal{B}[[b]] \sigma=\operatorname{true} \&\left(\sigma, \sigma^{\prime}\right) \in \mathcal{C}\left[\left[c_{0}\right]\right]\right\} \cup$

$\left\{\left(\sigma, \sigma^{\prime}\right) \mid \mathcal{B}[[b]] \sigma=\right.$ false $\left.\&\left(\sigma, \sigma^{\prime}\right) \in \mathcal{C}\left[\left[c_{1}\right]\right]\right\}$

$\mathcal{C}[[$ while $b$ do $c]]=f i x(\Gamma)$

with $\Gamma(\phi)=\left\{\left(\sigma, \sigma^{\prime}\right) \mid \mathcal{B}[[b]] \sigma=\operatorname{true} \&\left(\sigma, \sigma^{\prime}\right) \in \phi \circ \mathcal{C}[[c]]\right\} \cup$

$\left\{\left(\sigma, \sigma^{\prime}\right) \mid \mathcal{B}[[b]] \sigma=\right.$ false $\}$

$\left.\mathcal{C}\left[\left[c_{0} \| c_{1}\right]\right]=\mathcal{C}\left[\left[c_{0}\right]\right]\right\} \cup\left\{\mathcal{C}\left[\left[c_{1}\right]\right]\right.$

We can get the following propositions.

Proposition 3.10. $\mathcal{C}\left[\left[c_{0} \| c_{1}\right]\right]=\mathcal{C}\left[\left[c_{1} \| c_{0}\right]\right]$, for $c_{0}, c_{1} \in \mathbf{C o m}$.

Proof. By the definition of the denotation of $\|$, we can get:

$\mathcal{C}\left[\left[c_{0} \| c_{1}\right]\right]=\mathcal{C}\left[\left[c_{0}\right]\right] \cup \mathcal{C}\left[\left[c_{1}\right]\right]$

$\mathcal{C}\left[\left[c_{1} \| c_{0}\right]\right]=\mathcal{C}\left[\left[c_{1}\right]\right] \cup \mathcal{C}\left[\left[c_{0}\right]\right]$

So, $\mathcal{C}\left[\left[c_{0} \| c_{1}\right]\right]=\mathcal{C}\left[\left[c_{1} \| c_{0}\right]\right]$, for $c_{0}, c_{1} \in \mathbf{C o m}$, as desired.

Proposition 3.11. $\mathcal{C}\left[\left[\left(c_{0} \| c_{1}\right) \| c_{2}\right]\right]=\mathcal{C}\left[\left[c_{0} \|\left(c_{1} \| c_{2}\right)\right]\right]$, for $c_{0}, c_{1}, c_{2} \in \mathbf{C o m}$.

Proof. By the definition of the denotation of $\|$, we can get:

$\mathcal{C}\left[\left[\left(c_{0} \| c_{1}\right) \| c_{2}\right]\right]=\left(\mathcal{C}\left[\left[c_{0}\right]\right] \cup \mathcal{C}\left[\left[c_{1}\right]\right]\right) \cup \mathcal{C}\left[\left[c_{2}\right]\right]$

$\mathcal{C}\left[\left[c_{0} \|\left(c_{1} \| c_{2}\right)\right]\right]=\mathcal{C}\left[\left[c_{0}\right]\right] \cup\left(\mathcal{C}\left[\left[c_{1}\right]\right] \cup \mathcal{C}\left[\left[c_{2}\right]\right]\right)$

$\mathcal{C}\left[\left[\left(c_{0} \| c_{1}\right) \| c_{2}\right]\right]=\mathcal{C}\left[\left[c_{0} \|\left(c_{1} \| c_{2}\right)\right]\right]$, for $c_{0}, c_{1}, c_{2} \in \mathbf{C o m}$, as desired. 
Proposition 3.12. $\mathcal{C}\left[\left(\right.\right.$ if $b$ then $c_{0}$ else $\left.\left.\left.c_{1}\right) \| c_{2}\right]\right]=\mathcal{C}\left[\left[\right.\right.$ if $b$ then $c_{0} \| c_{2}$ else $\left.\left.c_{1} \| c_{2}\right]\right]$, for $c_{0}, c_{1}, c_{2} \in \mathbf{C o m}$

Proof. By the definition of the denotation of choice and $\|$, we can get:

$\mathcal{C}\left[\left[\left(\right.\right.\right.$ if $b$ then $c_{0}$ else $\left.\left.\left.c_{1}\right) \| c_{2}\right]\right]=\left\{\left(\sigma, \sigma^{\prime}\right) \mid \mathcal{B}[[b]] \sigma=\operatorname{true} \&\left(\sigma, \sigma^{\prime}\right) \in \mathcal{C}\left[\left[c_{0}\right]\right]\right\} \cup$

$\left\{\left(\sigma, \sigma^{\prime}\right) \mid \mathcal{B}[[b]] \sigma=\right.$ false $\left.\&\left(\sigma, \sigma^{\prime}\right) \in \mathcal{C}\left[\left[c_{1}\right]\right]\right\} \cup \mathcal{C}\left[\left[c_{2}\right]\right]$

$\mathcal{C}\left[\left[\right.\right.$ if $b$ then $c_{0} \| c_{2}$ else $\left.\left.c_{1} \| c_{2}\right]\right]=\left\{\left(\sigma, \sigma^{\prime}\right) \mid \mathcal{B}[[b]] \sigma=\operatorname{true} \&\left(\sigma, \sigma^{\prime}\right) \in \mathcal{C}\left[\left[c_{0}\right]\right] \cup \mathcal{C}\left[\left[c_{2}\right]\right]\right\} \cup$

$\left\{\left(\sigma, \sigma^{\prime}\right) \mid \mathcal{B}[[b]] \sigma=\right.$ false $\left.\&\left(\sigma, \sigma^{\prime}\right) \in \mathcal{C}\left[\left[c_{1}\right]\right] \cup \mathcal{C}\left[\left[c_{2}\right]\right]\right\}$

So, $\mathcal{C}\left[\left[\left(\right.\right.\right.$ if $b$ then $c_{0}$ else $\left.\left.\left.c_{1}\right) \| c_{2}\right]\right]=\mathcal{C}\left[\left[\right.\right.$ if $b$ then $c_{0} \| c_{2}$ else $\left.\left.c_{1} \| c_{2}\right]\right]$, for $c_{0}, c_{1}, c_{2} \in \mathbf{C o m}$, as desired.

Proposition 3.13. For $c_{0}, c_{1}, c_{2}, c_{3} \in \mathbf{C o m}$,

1. $\mathcal{C}\left[\left[\left(c_{0} ; c_{1}\right) \| c_{2}\right]\right]=\mathcal{C}\left[\left[\left(c_{0} \| c_{2}\right) ; c_{1}\right]\right] ;$

2. $\mathcal{C}\left[\left[\left(c_{0} ; c_{1}\right) \|\left(c_{2} ; c_{3}\right)\right]\right]=\mathcal{C}\left[\left[\left(c_{0} \| c_{2}\right) ;\left(c_{1} \| c_{3}\right)\right]\right]$.

Proof. (1)By the definition of the denotation of sequence and $\|$, we can get:

$\mathcal{C}\left[\left[\left(c_{0} ; c_{1}\right) \| c_{2}\right]\right]=\left(\mathcal{C}\left[\left[c_{1}\right]\right] \circ \mathcal{C}\left[\left[c_{0}\right]\right]\right) \cup \mathcal{C}\left[\left[c_{2}\right]\right]$

$\mathcal{C}\left[\left[\left(c_{0} \| c_{2}\right) ; c_{1}\right]\right]=\mathcal{C}\left[\left[c_{1}\right]\right] \circ\left(\mathcal{C}\left[\left[c_{0}\right] \cup \mathcal{C}\left[\left[c_{2}\right]\right]\right)\right.$

So, $\mathcal{C}\left[\left[\left(c_{0} ; c_{1}\right) \| c_{2}\right]\right]=\mathcal{C}\left[\left[\left(c_{0} \| c_{2}\right) ; c_{1}\right]\right]$, as desired.

(1)By the definition of the denotation of sequence and $\|$, we can get:

$\mathcal{C}\left[\left[\left(c_{0} ; c_{1}\right) \|\left(c_{2} ; c_{3}\right)\right]\right]=\left(\mathcal{C}\left[\left[c_{1}\right]\right] \circ \mathcal{C}\left[\left[c_{0}\right]\right]\right) \cup\left(\mathcal{C}\left[\left[c_{3}\right]\right] \circ \mathcal{C}\left[\left[c_{2}\right]\right]\right.$

$\mathcal{C}\left[\left[\left(c_{0} \| c_{2}\right) ;\left(c_{1} \| c_{3}\right)\right]\right]=\left(\mathcal{C}\left[\left[c_{1}\right]\right] \cup \mathcal{C}\left[\left[c_{3}\right]\right]\right) \circ\left(\mathcal{C}\left[\left[c_{2}\right]\right] \cup \mathcal{C}\left[\left[c_{0}\right]\right]\right.$

So, $\mathcal{C}\left[\left[\left(c_{0} ; c_{1}\right) \|\left(c_{2} ; c_{3}\right)\right]\right]=\mathcal{C}\left[\left[\left(c_{0} \| c_{2}\right) ;\left(c_{1} \| c_{3}\right)\right]\right]$, as desired.

Proposition 3.14. $\mathcal{C}[[c \| \mathbf{s k i p}]]=\mathcal{C}[[c]]$, for $c \in \mathbf{C o m}$.

Proof. By the definition of the denotation of skip and $\|$, we can get:

$\mathcal{C}[[c \|$ skip $]]=\mathcal{C}[[c]] \cup \mathcal{C}[[$ skip $]]$

So, $\mathcal{C} \llbracket c \|$ skip $\rrbracket=\mathcal{C} \llbracket c \rrbracket$, for $c \in \mathbf{C o m}$, as desired.

Lemma 3.15. For $c_{0}, c_{1} \in \mathbf{C o m}$,

1. $c_{0}\left\|c_{1} \sim c_{0}\right\|\left(\right.$ skip; $\left.c_{1}\right) \sim c_{0} ; c_{1}$;

2. $c_{0} \| c_{1} \sim\left(\right.$ skip; $\left.c_{0}\right) \| c_{1} \sim c_{1} ; c_{0}$.

Proof. It is obvious by Proposition 3.13 and 3.15 . 


\subsection{Relations between Operational and Denotational Semantics}

The operational and denotational semantics still agree on the evaluation of Aexp and Bexp, we do not repeat any more, please refer to [21] for details. We will prove the agreement of the case Com as follows.

Lemma 3.16. For all commands $c$ and states $\sigma, \sigma^{\prime}$,

$$
\langle c, \sigma\rangle \rightarrow \sigma^{\prime} \Rightarrow\left(\sigma, \sigma^{\prime}\right) \in \mathcal{C}[[c]]
$$

Proof. We will use rule-induction on the operational semantics of commands. For $c \in \mathbf{C o m}$ and $\sigma, \sigma^{\prime} \in \Sigma$, define

$$
P\left(c, \sigma, \sigma^{\prime}\right) \Leftrightarrow_{\text {def }}\left(\sigma, \sigma^{\prime}\right) \in \mathcal{C}[[c]]
$$

We will show $P$ is closed under the rules for the execution of commands, and we will only prove the new case of $\|$, other commands please refer to [21] for details.

Recall the transition rules of $\|$ are:

$$
\frac{\left\langle c_{1}, \sigma\right\rangle \rightarrow \sigma^{\prime} \quad\left\langle c_{0}, \sigma\right\rangle \rightarrow \sigma^{\prime \prime}}{\left\langle c_{0} \| c_{1}, \sigma\right\rangle \rightarrow \sigma^{\prime} \uplus \sigma^{\prime \prime}}
$$

Assume that

$$
\left\langle c_{0}, \sigma\right\rangle \rightarrow \sigma^{\prime} \& P\left(c_{0}, \sigma, \sigma^{\prime}\right) \&\left\langle c_{1}, \sigma\right\rangle \rightarrow \sigma^{\prime \prime} \& P\left(c_{1}, \sigma, \sigma^{\prime \prime}\right)
$$

From the meaning of $P$, we can get that

$$
\mathcal{C}\left[\left[c_{0}\right]\right] \sigma=\sigma^{\prime} \text { and } \mathcal{C}\left[\left[c_{1}\right]\right] \sigma=\sigma^{\prime \prime}
$$

We can get

$$
\mathcal{C}\left[\left[c_{0} \| c_{1}\right]\right] \sigma=\sigma^{\prime} \uplus \sigma^{\prime \prime}
$$

which means that $P\left(c_{0} \| c_{1}, \sigma, \sigma^{\prime} \uplus \sigma^{\prime \prime}\right)$ holds for the consequence of the rule, and is closed under this rule.

Theorem 3.17. For all commands $c$ and states $\sigma, \sigma^{\prime}$,

$$
\mathcal{C}[[c]]=\left\{\left(\sigma, \sigma^{\prime}\right) \mid\langle c, \sigma\rangle \rightarrow \sigma^{\prime}\right\}
$$

Proof. Lemma 3.16 gives the $\Leftarrow$ direction of proof, we only need to prove

$$
\left(\sigma, \sigma^{\prime}\right) \in \mathcal{C}[[c]] \Rightarrow\langle c, \sigma\rangle \rightarrow \sigma^{\prime}
$$

It is sufficient to induct on the structure of command $c$, we only prove the new case of $c \equiv c_{0} \| c_{1}$, other cases please refer to [21] for details. 
Suppose $\left(\sigma, \sigma^{\prime} \uplus \sigma^{\prime \prime}\right) \in \mathcal{C}[[c]]$. Then there are some states, such that $\left(\sigma, \sigma^{\prime}\right) \in \mathcal{C}\left[\left[c_{0}\right]\right],\left(\sigma, \sigma^{\prime \prime}\right) \in$ $\mathcal{C}\left[\left[c_{1}\right]\right]$. By the hypothesis of $c_{0}, c_{1}$, we get

$$
\left\langle c_{0}, \sigma\right\rangle \rightarrow \sigma^{\prime} \text { and }\left\langle c_{1}, \sigma\right\rangle \rightarrow \sigma^{\prime \prime}
$$

So, $\left\langle c_{0} \| c_{1}, \sigma\right\rangle \rightarrow \sigma^{\prime} \uplus \sigma^{\prime \prime}$, as desired.

\subsection{Axiomatic Semantics}

In this section, we give an axiomatic semantics for PPL by extending the Hoare rules with parallelism.

\subsubsection{Extended Hoare Rules for Parallelism}

PPL should be extended to support assertion.

For Aexp, it should be extended to:

$$
a::=n \quad|\quad X \quad| \quad i \quad\left|\quad a_{0}+a_{1}\right| \begin{array}{llll}
a_{0}-a_{1} & \mid & a_{0} \times a_{1}
\end{array}
$$

where $i$ ranges over integer variables, Intvar.

For Bexp, it should be extended to support boolean assertion:

$$
A::=\text { true } \mid \text { false }\left|a_{0}=a_{1}\right| a_{0} \leq a_{1}|\neg A| A_{0} \wedge A_{1}\left|A_{0} \vee A_{1}\right| A_{0} \Rightarrow A_{1}|\forall i . A| \exists i . A
$$

And the formation rule of Com is maintained:

$$
c::=\text { skip }|X:=a \quad| \quad c_{0} ; c_{1} \quad \mid \quad \text { if } b \text { then } c_{0} \text { else } c_{1} \quad \mid \quad \text { while } b \text { do } c \quad \mid \quad c_{0} \| c_{1}
$$

Note that, Com contains a parallel composition $\|$.

The denotational semantics should also contain an interpretation $I$.

The full extended Hoare rules are as follow.

Rule for skip:

$$
\{A\} \text { skip }\{A\}
$$

Rule for assignments:

$$
\{B[a / X]\} X:=a\{B\}
$$

Rule for sequencing:

$$
\frac{\{A\} c_{0}\{C\} \quad\{C\} c_{1}\{B\}}{\{A\} c_{0} ; c_{1}\{B\}}
$$


Rule for conditionals:

$$
\frac{\{A \wedge b\} c_{0}\{B\} \quad\{A \wedge \neg b\} c_{1}\{B\}}{\{A\} \text { if } b \text { then } c_{0} \text { else } c_{1}\{B\}}
$$

Rule for while loops:

$$
\frac{\{A \wedge b\} c\{A\}}{\{A\} \text { while } b \text { do } c\{A \wedge \neg b\}}
$$

Rule for consequence:

$$
\frac{\vDash\left(A \Rightarrow A^{\prime}\right) \quad\left\{A^{\prime}\right\} c\left\{B^{\prime}\right\} \quad \vDash\left(B^{\prime} \Rightarrow B\right)}{\{A\} c\{B\}}
$$

Rule for parallelism:

$$
\frac{\{A\} c_{0}\{C\} \quad\{C\} c_{1}\{B\} \quad\{A\} c_{1}\{D\} \quad\{D\} c_{0}\{B\}}{\{A\} c_{0} \| c_{1}\{B\}}
$$

\subsubsection{Soundness of The Extended Hoare Rules}

We can prove that each rule is sound by the following soundness theorem.

Theorem 3.18. Let $\{A\} c\{B\}$ be a partial correctness assertion, if $\vdash\{A\} c\{B\}$, then $\vDash\{A\} c\{B\}$.

Proof. It is sufficient to induct on the rule to prove each rule is valid. We only prove the new case of $\|$ rule, other cases please refer to [21] for details.

Assume that $\vDash\{A\} c_{0}\{C\}$ and $\vDash\{C\} c_{1}\{B\}$, and $\vDash\{A\} c_{1}\{D\}$ and $\vDash\{D\} c_{0}\{B\}$. Let $I$ be an interpretation. Suppose $\sigma \vDash^{I} A$. Then $\mathcal{C}\left[\left[c_{0}\right]\right] \sigma \vDash^{I} C$ and $\mathcal{C}\left[\left[c_{1}\right]\right]\left(\mathcal{C}\left[\left[c_{0}\right]\right] \sigma\right) \vDash^{I} B$, and $\mathcal{C}\left[\left[c_{1}\right]\right] \sigma \vDash^{I} D$ and $\mathcal{C}\left[\left[c_{0}\right]\right]\left(\mathcal{C}\left[\left[c_{1}\right]\right] \sigma\right) \vDash^{I} B$. Hence, $\vDash\{A\} c_{0} \| c_{1}\{B\}$, as desired.

\subsubsection{Completeness of The Extended Hoare Rules}

Gödel's Incompleteness Theorem implies that the extended Hoare rules are incomplete. We prove the relative completeness in the sense of Cook.

Theorem 3.19. PPL extended with assertion is expressive.

Proof. It is sufficient to induct on the structure of command $c$, such that for all assertions $B$ there is an assertion $w[[c, B]]$, for all interpretations $I$

$$
w p^{I}[[c, B]]=w[[c, B]]^{I}
$$

We only prove the new case of parallelism $c \equiv c_{0} \| c_{1}$, other cases please refer to [21] for details. Inductively define $w\left[\left[c_{0} \| c_{1}, B\right]\right] \equiv w\left[\left[c_{0}, w\left[\left[c_{1}, B\right]\right]\right]\right]$ and $w\left[\left[c_{0} \| c_{1}, B\right]\right] \equiv w\left[\left[c_{1}, w\left[\left[c_{0}, B\right]\right]\right]\right.$. Then, for $\sigma \in \Sigma$ and any interpretation $I$, 
$\sigma \in w p^{I}\left[\left[c_{0} \| c_{1}, B\right]\right]$ iff $\mathcal{C}\left[\left[c_{0} \| c_{1}\right]\right] \sigma \vDash^{I} B$

iff $\mathcal{C}\left[\left[c_{1}\right]\right]\left(\mathcal{C}\left[\left[c_{0}\right]\right] \sigma\right) \vDash^{I} B$ and $\mathcal{C}\left[\left[c_{0}\right]\right]\left(\mathcal{C}\left[\left[c_{1}\right]\right] \sigma\right) \vDash^{I} B$

iff $\mathcal{C}\left[\left[c_{0}\right]\right] \sigma \vDash^{I} w\left[\left[c_{1}, B\right]\right]$ and $\mathcal{C}\left[\left[c_{1}\right]\right] \sigma \vDash^{I} w\left[\left[c_{0}, B\right]\right]$

iff $\sigma \vDash^{I} w\left[\left[c_{0}, w\left[\left[c_{1}, B\right]\right]\right]\right]$ and $\sigma \vDash^{I} w\left[\left[c_{1}, w\left[\left[c_{0}, B\right]\right]\right]\right]$

iff $\sigma \vDash^{I} w\left[\left[c_{0} \| c_{1}, B\right]\right]$.

Lemma 3.20. For $c \in \mathbf{C o m}$ and $B$ is an assertion, let $w[[c, B]]$ be an assertion expressing the weakest precondition with $w[[c, B]]^{I}=w p^{I}[[c, B]]$. Then

$$
\vdash\{w[[c, B]]\} c\{B\}
$$

Proof. It suffices to induct on the structure of commands $c$, we only prove the new case of parallelism $c \equiv c_{0} \| c_{1}$, other cases please refer to [21] for details.

For $\sigma \in \Sigma$ and any interpretation $I$,

$\sigma \vDash^{I} w\left[\left[c_{0} \| c_{1}, B\right]\right]$ iff $\mathcal{C}\left[\left[c_{0} \| c_{1}\right]\right] \sigma \vDash^{I} B$

iff $\mathcal{C}\left[\left[c_{1}\right]\right]\left(\mathcal{C}\left[\left[c_{0}\right]\right] \sigma\right) \vDash^{I} B$ and $\mathcal{C}\left[\left[c_{0}\right]\right]\left(\mathcal{C}\left[\left[c_{1}\right]\right] \sigma\right) \vDash^{I} B$

iff $\mathcal{C}\left[\left[c_{0}\right]\right] \sigma \vDash^{I} w\left[\left[c_{1}, B\right]\right]$ and $\mathcal{C}\left[\left[c_{1}\right]\right] \sigma \vDash^{I} w\left[\left[c_{0}, B\right]\right]$

iff $\sigma \vDash^{I} w\left[\left[c_{0}, w\left[\left[c_{1}, B\right]\right]\right]\right]$ and $\sigma \vDash^{I} w\left[\left[c_{1}, w\left[\left[c_{0}, B\right]\right]\right]\right]$.

We get $\vdash\left\{w\left[\left[c_{0}, w\left[\left[c_{1}, B\right]\right]\right]\right]\right\} c_{0} \| c_{1}\{B\}$ and $\vdash\left\{w\left[\left[c_{1}, w\left[\left[c_{0}, B\right]\right]\right]\right]\right\} c_{0} \| c_{1}\{B\}$.

Hence, by the consequence rule, we obtain

$$
\vdash\left\{w\left[\left[c_{0} \| c_{1}, B\right]\right]\right\} c_{0} \| c_{1}\{B\}
$$

Theorem 3.21. For any partial correctness assertion $\{A\} c\{B\}$, if $\vDash\{A\} c\{B\}$, then $\vdash\{A\} c\{B\}$.

Proof. Suppose $\vDash\{A\} c\{B\}$, then $\vdash\{w[[c, B]]\} c\{B\}$ where $w[[c, B]]^{I}=w p^{I}[[c, B]]$ for any interpretation $I$ (by the above Lemma). Hence, $\vDash(A \Rightarrow w[[c, B]])$, we obtain $\vdash\{A\} c\{B\}$.

\subsection{Non-determinism}

The guarded commands can make the use of non-determinism more rigorous. To provide each command with a conditional guard, it is useful to eliminate the uncontrolled non-determinism.

The syntax of guarded commands are also composed of Aexp, Bexp and Com, and the syntax of Aexp and Bexp are the same as those of PPL in section 3.1. And the formation rules for the command $c$ and guarded commands $g c$ are as follow.

$$
\begin{gathered}
c::=\text { skip } \mid \text { abort }|X:=a \quad| \quad c_{0} ; c_{1} \mid \text { if } g c \text { fi } \mid \text { do } g c \text { od } \\
\qquad c::=b \rightarrow c \quad \mid \quad g c_{0} \square g c_{1}
\end{gathered}
$$


where $g c_{0} \square g c_{1}$ is the alternative construct of $g c_{0}$ and $g c_{1}$.

The operational rules of commands:

$$
\begin{gathered}
\langle\text { skip }, \sigma\rangle \rightarrow \sigma \\
\frac{\langle a, \sigma\rangle \rightarrow n}{\langle X:=a, \sigma\rangle \rightarrow \sigma[n / X]} \\
\frac{\left\langle c_{0}, \sigma\right\rangle \rightarrow \sigma^{\prime}}{\left\langle c_{0} ; c_{1}, \sigma\right\rangle \rightarrow\left\langle c_{1}, \sigma^{\prime}\right\rangle} \frac{\left\langle c_{0}, \sigma\right\rangle \rightarrow\left\langle c_{0}^{\prime}, \sigma^{\prime}\right\rangle}{\left\langle c_{0} ; c_{1}, \sigma\right\rangle \rightarrow\left\langle c_{0}^{\prime} ; c_{1}, \sigma^{\prime}\right\rangle} \\
\frac{\langle g c, \sigma\rangle \rightarrow\left\langle c, \sigma^{\prime}\right\rangle}{\langle\text { if } g c \text { fi, } \sigma\rangle \rightarrow\left\langle c, \sigma^{\prime}\right\rangle} \\
\frac{\langle g c, \sigma\rangle \rightarrow \text { fail }}{\langle\text { do } g c \text { od }, \sigma\rangle \rightarrow \sigma} \quad \frac{\langle g c, \sigma\rangle \rightarrow\left\langle c, \sigma^{\prime}\right\rangle}{\langle\text { do } g c \text { od }, \sigma\rangle \rightarrow\left\langle c ; \text { do } g c \text { od }, \sigma^{\prime}\right\rangle}
\end{gathered}
$$

The operational rules of guarded commands:

$$
\begin{gathered}
\frac{\langle b, \sigma\rangle \rightarrow \text { true }}{\langle b \rightarrow c, \sigma\rangle \rightarrow\langle c, \sigma\rangle} \\
\frac{\left\langle g c_{0}, \sigma\right\rangle \rightarrow\left\langle c, \sigma^{\prime}\right\rangle}{\left\langle g c_{0} \square g c_{1}, \sigma\right\rangle \rightarrow\left\langle c, \sigma^{\prime}\right\rangle} \quad \frac{\left\langle g c_{1}, \sigma\right\rangle \rightarrow\left\langle c, \sigma^{\prime}\right\rangle}{\left\langle g c_{0} \square g c_{1}, \sigma\right\rangle \rightarrow\left\langle c, \sigma^{\prime}\right\rangle} \\
\frac{\langle b, \sigma\rangle \rightarrow \text { false }}{\langle b \rightarrow c, \sigma\rangle \rightarrow \text { fail }} \frac{\left\langle g c_{0}, \sigma\right\rangle \rightarrow \text { false } \quad\left\langle g c_{1}, \sigma\right\rangle \rightarrow \text { false }}{\left\langle g c_{0} \square g c_{1}, \sigma\right\rangle \rightarrow \text { fail }}
\end{gathered}
$$

\subsection{Communications}

In this section, we extend communicating processes with the support for true concurrency.

The syntax of PPL are also composed of Aexp, Bexp, the names of communication channels $\alpha, \beta, \gamma \in \mathbf{C h a n}$, and Com, and the syntax of Aexp and Bexp are the same as those of PPL in section 3.1. And the formation rules for the command $c$ and guarded commands $g c$ are as follow.

$$
\begin{gathered}
c::=\operatorname{skip} \mid \text { abort }|X:=a \quad| \alpha ? X|\alpha ! a| c_{0} ; c_{1} \mid \text { if } g c \text { fi|do } g c \text { od }\left|c_{0} \| c_{1}\right| c \backslash \alpha \\
g c::=b \rightarrow c \quad|\quad b \wedge \alpha ? X \rightarrow c \quad| \quad b \wedge \alpha ! a \quad \mid \quad g c_{0} \square g c_{1}
\end{gathered}
$$

where $g c_{0} \square g c_{1}$ is the alternative construct of $g c_{0}$ and $g c_{1}$.

The operational rules of commands:

$$
\langle\text { skip, } \sigma\rangle \rightarrow \sigma
$$




$$
\begin{aligned}
& \frac{\langle a, \sigma\rangle \rightarrow n}{\langle X:=a, \sigma\rangle \rightarrow \sigma[n / X]} \\
& \langle\alpha ? X, \sigma\rangle \stackrel{\alpha ? n}{\longrightarrow} \sigma[n / X] \\
& \frac{\langle a, \sigma\rangle \rightarrow n}{\langle\alpha ! a, \sigma\rangle \stackrel{\alpha ! n}{\longrightarrow} \sigma} \\
& \frac{\left\langle c_{0}, \sigma\right\rangle \rightarrow \sigma^{\prime}}{\left\langle c_{0} ; c_{1}, \sigma\right\rangle \rightarrow\left\langle c_{1}, \sigma^{\prime}\right\rangle} \quad \frac{\left\langle c_{0}, \sigma\right\rangle \rightarrow\left\langle c_{0}^{\prime}, \sigma^{\prime}\right\rangle}{\left\langle c_{0} ; c_{1}, \sigma\right\rangle \rightarrow\left\langle c_{0}^{\prime} ; c_{1}, \sigma^{\prime}\right\rangle} \\
& \frac{\langle g c, \sigma\rangle \rightarrow\left\langle c, \sigma^{\prime}\right\rangle}{\langle\text { if } g c \text { fi, } \sigma\rangle \rightarrow\left\langle c, \sigma^{\prime}\right\rangle} \\
& \frac{\langle g c, \sigma\rangle \rightarrow \text { fail }}{\langle\operatorname{dog} g c \text { od, } \sigma\rangle \rightarrow \sigma} \quad \frac{\langle g c, \sigma\rangle \rightarrow\left\langle c, \sigma^{\prime}\right\rangle}{\langle\operatorname{dog} g c \text { od, } \sigma\rangle \rightarrow\left\langle c ; \operatorname{dog} g c \text { od, } \sigma^{\prime}\right\rangle} \\
& \frac{\left\langle c_{0}, \sigma\right\rangle \stackrel{\lambda}{\rightarrow}\left\langle c_{0}^{\prime}, \sigma^{\prime}\right\rangle \quad c_{0} \% c_{1}}{\left\langle c_{0} \| c_{1}, \sigma\right\rangle \stackrel{\lambda}{\rightarrow}\left\langle c_{0}^{\prime} \| c_{1}, \sigma^{\prime}\right\rangle} \\
& \frac{\left\langle c_{1}, \sigma\right\rangle \stackrel{\lambda}{\rightarrow}\left\langle c_{1}^{\prime}, \sigma^{\prime}\right\rangle \quad c_{0} \% c_{1}}{\left\langle c_{0} \| c_{1}, \sigma\right\rangle \stackrel{\lambda}{\rightarrow}\left\langle c_{0} \| c_{1}^{\prime}, \sigma^{\prime}\right\rangle} \\
& \frac{\left\langle c_{0}, \sigma\right\rangle \stackrel{\lambda_{1}}{\longrightarrow}\left\langle c_{0}^{\prime}, \sigma^{\prime}\right\rangle \quad\left\langle c_{1}, \sigma\right\rangle \stackrel{\lambda_{2}}{\longrightarrow}\left\langle c_{1}^{\prime}, \sigma^{\prime \prime}\right\rangle}{\left\langle c_{0} \| c_{1}, \sigma\right\rangle \stackrel{\left\{\lambda_{1}, \lambda_{2}\right\}}{\longrightarrow}\left\langle c_{0}^{\prime} \| c_{1}^{\prime}, \sigma^{\prime} \uplus \sigma^{\prime \prime}\right\rangle} \\
& \frac{\left\langle c_{0}, \sigma\right\rangle \stackrel{\alpha ! n}{\longrightarrow}\left\langle c_{0}^{\prime}, \sigma\right\rangle\left\langle c_{1}, \sigma\right\rangle \stackrel{\alpha ? n}{\longrightarrow}\left\langle c_{1}^{\prime}, \sigma^{\prime}\right\rangle}{\left\langle c_{0} \| c_{1}, \sigma\right\rangle \stackrel{\gamma_{\alpha}(n)}{\longrightarrow}\left\langle c_{0}^{\prime} \| c_{1}^{\prime}, \sigma^{\prime}\right\rangle} \\
& \frac{\left\langle c_{0}, \sigma\right\rangle \stackrel{\alpha ? n}{\longrightarrow}\left\langle c_{0}^{\prime}, \sigma^{\prime}\right\rangle\left\langle c_{1}, \sigma\right\rangle \stackrel{\alpha ! n}{\longrightarrow}\left\langle c_{1}^{\prime}, \sigma\right\rangle}{\left\langle c_{0} \| c_{1}, \sigma\right\rangle \stackrel{\gamma_{\alpha}(n)}{\longrightarrow}\left\langle c_{0}^{\prime} \| c_{1}^{\prime}, \sigma^{\prime}\right\rangle} \\
& \frac{\langle c, \sigma\rangle \stackrel{\lambda}{\rightarrow}\left\langle c^{\prime}, \sigma^{\prime}\right\rangle}{\langle c \backslash \alpha, \sigma\rangle \stackrel{\lambda}{\rightarrow}\left\langle c^{\prime} \backslash \alpha, \sigma^{\prime}\right\rangle} \text { if } \lambda \equiv \alpha ? n \text { and } \lambda \equiv \alpha ! n \text { do not hold }
\end{aligned}
$$

Where $c_{0} \% c_{1}$ denotes that $c_{0}$ and $c_{1}$ are in race condition.

The operational rules of guarded commands:

$$
\frac{\langle b, \sigma\rangle \rightarrow \text { true }}{\langle b \rightarrow c, \sigma\rangle \rightarrow\langle c, \sigma\rangle}
$$




$$
\begin{gathered}
\frac{\left\langle g c_{0}, \sigma\right\rangle \rightarrow\left\langle c, \sigma^{\prime}\right\rangle}{\left\langle g c_{0} \square g c_{1}, \sigma\right\rangle \rightarrow\left\langle c, \sigma^{\prime}\right\rangle} \quad \frac{\left\langle g c_{1}, \sigma\right\rangle \rightarrow\left\langle c, \sigma^{\prime}\right\rangle}{\left\langle g c_{0} \square g c_{1}, \sigma\right\rangle \rightarrow\left\langle c, \sigma^{\prime}\right\rangle} \\
\frac{\langle b, \sigma\rangle \rightarrow \text { false }}{\langle b \rightarrow c, \sigma\rangle \rightarrow \text { fail }} \quad \frac{\left\langle g c_{0}, \sigma\right\rangle \rightarrow \text { false } \quad\left\langle g c_{1}, \sigma\right\rangle \rightarrow \text { false }}{\left\langle g c_{0} \square g c_{1}, \sigma\right\rangle \rightarrow \text { fail }} \\
\frac{\langle b, \sigma\rangle \rightarrow \text { false }}{\langle b \wedge \alpha ? X \rightarrow c, \sigma\rangle \rightarrow \text { fail }} \\
\frac{\langle b, \sigma\rangle \rightarrow \text { false }}{\langle b \wedge \alpha ! X \rightarrow c, \sigma\rangle \rightarrow \text { fail }} \\
\frac{\langle b, \sigma\rangle \rightarrow \text { true }}{\langle b \wedge \alpha ? X \rightarrow c, \sigma\rangle \stackrel{\alpha ? n}{\longrightarrow}\langle c, \sigma[n / X]\rangle} \\
\frac{\langle b, \sigma\rangle \rightarrow \text { true }\langle a, \sigma\rangle \rightarrow n}{\langle b \wedge \alpha ! a \rightarrow c, \sigma\rangle \stackrel{\alpha ! n}{\longrightarrow}\langle c, \sigma\rangle}
\end{gathered}
$$

Note that, for true concurrency, we can see that communications, conflictions, and race conditions are solved as follows.

1. Communication is explicitly supported in PPL, the two communicating commands $\alpha$ ? $X$ and $\alpha ! X$ will merge to one communication command $\gamma_{\alpha}(X)$, and the unstructured communication will be eliminated;

2. Since each command is with a guard, the conflictions among actions can be achieved by set the commands with exclusive guards;

3. As the operational rules state, the actions in parallel in race condition must be executed sequentially and will cause the non-deterministic execution order. Though the execution order is non-deterministic, by setting appropriate guards to the parallel commands, the final execution configuration can be deterministic.

We can get the following propositions. Where $\sim$ is an equivalence relation on commands by the definition, where $\Sigma$ is the set of states:

Definition 3.22 (Equivalence of operational semantics for commands). $c_{0} \sim c_{1}$ iff $\forall \sigma, \sigma^{\prime} \epsilon$ $\Sigma,\left\langle c_{0}, \sigma\right\rangle \rightarrow \sigma^{\prime} \Leftrightarrow\left\langle c_{1}, \sigma\right\rangle \rightarrow \sigma^{\prime}$

Proposition 3.23. $c_{0}\left\|c_{1} \sim c_{1}\right\| c_{0}$, for $c_{0}, c_{1} \in \mathbf{C o m}$.

Proof. By use of the transition rules of $\|$, we can get the following derivations of $c_{0} \| c_{1}$ for $\forall \sigma \in \Sigma:$

$$
\frac{\left\langle c_{0}, \sigma\right\rangle \stackrel{c_{0}}{\longrightarrow} \sigma^{\prime \prime} \quad\left\langle c_{1}, \sigma^{\prime \prime}\right\rangle \stackrel{c_{1}}{\longrightarrow} \sigma^{\prime}}{\left\langle c_{0} \| c_{1}, \sigma\right\rangle \stackrel{c_{0} ; c_{1}}{\longrightarrow} \sigma^{\prime}}
$$




$$
\begin{gathered}
\frac{\left.\left\langle c_{1}, \sigma\right\rangle \stackrel{c_{1}}{\rightarrow} \sigma^{\prime \prime \prime} \quad\left\langle c_{0}, \sigma^{\prime \prime \prime}\right\rangle \stackrel{(}{\rightarrow} c_{0}\right) \sigma^{\prime}}{\left.\left\langle c_{0} \| c_{1}, \sigma\right\rangle \stackrel{(}{\rightarrow} c_{1} ; c_{0}\right) \sigma^{\prime}} \\
\frac{\left\langle c_{1}, \sigma\right\rangle \stackrel{c_{1}}{\longrightarrow} \sigma^{\prime} \quad\left\langle c_{0}, \sigma\right\rangle \stackrel{c_{0}}{\longrightarrow} \sigma^{\prime \prime}}{\left\langle c_{0} \| c_{1}, \sigma\right\rangle \stackrel{\left\{c_{0}, c_{1}\right\}}{\longrightarrow} \sigma^{\prime} \uplus \sigma^{\prime \prime}}
\end{gathered}
$$

And we can get the following derivations of $c_{1} \| c_{0}$ for $\forall \sigma \in \Sigma$ :

$$
\begin{gathered}
\frac{\left\langle c_{1}, \sigma\right\rangle \stackrel{c_{1}}{\longrightarrow} \sigma^{\prime \prime \prime} \quad\left\langle c_{0}, \sigma^{\prime \prime \prime}\right\rangle \stackrel{c_{0}}{\longrightarrow} \sigma^{\prime}}{\left\langle c_{1} \| c_{0}, \sigma\right\rangle \stackrel{c_{1} ; c_{0}}{\longrightarrow} \sigma^{\prime}} \\
\frac{\left\langle c_{0}, \sigma\right\rangle \stackrel{c_{0}}{\longrightarrow} \sigma^{\prime \prime} \quad\left\langle c_{1}, \sigma^{\prime \prime}\right\rangle \stackrel{c_{1}}{\longrightarrow} \sigma^{\prime}}{\left\langle c_{1} \| c_{0}, \sigma\right\rangle \stackrel{c_{0} ; c_{1}}{\longrightarrow} \sigma^{\prime}} \\
\frac{\left\langle c_{0}, \sigma\right\rangle \stackrel{c_{0}}{\longrightarrow} \sigma^{\prime}\left\langle c_{1}, \sigma\right\rangle \stackrel{c_{1}}{\longrightarrow} \sigma^{\prime \prime}}{\left\langle c_{1} \| c_{0}, \sigma\right\rangle \stackrel{\left\{c_{0}, c_{1}\right\}}{\longrightarrow} \sigma^{\prime} \uplus \sigma^{\prime \prime}}
\end{gathered}
$$

So, it is obvious that $c_{0}\left\|c_{1} \sim c_{1}\right\| c_{0}$, for $c_{0}, c_{1} \in \mathbf{C o m}$, as desired.

Proposition 3.24. $\left(c_{0} \| c_{1}\right)\left\|c_{2} \sim c_{0}\right\|\left(c_{1} \| c_{2}\right)$, for $c_{0}, c_{1}, c_{2} \in \mathbf{C o m}$.

Proof. By use of the transition rules of $\|$, we can get the following derivations of $\left(c_{0} \| c_{1}\right) \| c_{2}$ for $\forall \sigma \in \Sigma$ :

$$
\frac{\left\langle c_{0}, \sigma\right\rangle \stackrel{c_{0}}{\longrightarrow} \sigma^{\prime} \quad\left\langle c_{1}, \sigma\right\rangle \stackrel{c_{1}}{\longrightarrow} \sigma^{\prime \prime} \quad\left\langle c_{2}, \sigma\right\rangle \stackrel{c_{2}}{\longrightarrow} \sigma^{\prime \prime \prime}}{\left\langle\left(c_{0} \| c_{1}\right) \| c_{2}, \sigma\right\rangle \stackrel{\left\{c_{0}, c_{1}, c_{2}\right\}}{\longrightarrow} \sigma^{\prime} \uplus \sigma^{\prime \prime} \uplus \sigma^{\prime \prime \prime}}
$$

And we can get the following derivations of $c_{0} \|\left(c_{1} \| c_{2}\right)$ for $\forall \sigma \in \Sigma$ :

$$
\frac{\left\langle c_{0}, \sigma\right\rangle \stackrel{c_{0}}{\longrightarrow} \sigma^{\prime} \quad\left\langle c_{1}, \sigma\right\rangle \stackrel{c_{1}}{\longrightarrow} \sigma^{\prime \prime} \quad\left\langle c_{2}, \sigma\right\rangle \stackrel{c_{2}}{\longrightarrow} \sigma^{\prime \prime \prime}}{\left\langle c_{0} \|\left(c_{1} \| c_{2}\right), \sigma\right\rangle \stackrel{\left\{c_{0}, c_{1}, c_{2}\right\}}{\longrightarrow} \sigma^{\prime} \uplus \sigma^{\prime \prime} \uplus \sigma^{\prime \prime \prime}}
$$

So, it is obvious that $\left(c_{0} \| c_{1}\right)\left\|c_{2} \sim c_{0}\right\|\left(c_{1} \| c_{2}\right)$, for $c_{0}, c_{1}, c_{2} \in \mathbf{C o m}$, as desired.

For the case of the parallel commands in race condition, we omit it.

Proposition 3.25. For $c_{0}, c_{1}, c_{2}, c_{3} \in \mathbf{C o m}$,

1. $\left(c_{0} ; c_{1}\right) \| c_{2} \sim\left(c_{0} \| c_{2}\right) ; c_{1}$;

2. $\left(c_{0} ; c_{1}\right) \|\left(c_{2} ; c_{3}\right) \sim\left(c_{0} \| c_{2}\right) ;\left(c_{1} \| c_{3}\right)$. 
Proof. (1) By use of the transition rules of sequence and $\|$, we can get the following derivations of $\left(c_{0} ; c_{1}\right) \| c_{2}$ :

$$
\frac{\left\langle c_{0}, \sigma\right\rangle \stackrel{c_{0}}{\longrightarrow} \sigma^{\prime}\left\langle c_{2}, \sigma\right\rangle \stackrel{c_{2}}{\longrightarrow} \sigma^{\prime \prime}}{\left\langle\left(c_{0} ; c_{1}\right) \| c_{2}, \sigma\right\rangle \stackrel{\left\{c_{0}, c_{2}\right\}}{\longrightarrow}\left\langle c_{1}, \sigma^{\prime} \uplus \sigma^{\prime \prime}\right\rangle}
$$

And we can get the following derivations of $\left(c_{0} \| c_{2}\right) ; c_{1}$ :

$$
\frac{\left\langle c_{0}, \sigma\right\rangle \stackrel{c_{0}}{\longrightarrow} \sigma^{\prime}\left\langle c_{2}, \sigma\right\rangle \stackrel{c_{2}}{\longrightarrow} \sigma^{\prime \prime}}{\left\langle\left(c_{0} \| c_{2}\right) ; c_{1}, \sigma\right\rangle \stackrel{\left\{c_{0}, c_{2}\right\}}{\longrightarrow}\left\langle c_{1}, \sigma^{\prime} \uplus \sigma^{\prime \prime}\right\rangle}
$$

So, it is obvious that $\left(c_{0} ; c_{1}\right) \| c_{2} \sim\left(c_{0} \| c_{2}\right) ; c_{1}$, for $c_{0}, c_{1}, c_{2} \in \mathbf{C o m}$, as desired.

(2) By use of the transition rules of sequence and $\|$, we can get the following derivations of $\left(c_{0} ; c_{1}\right) \|\left(c_{2} ; c_{3}\right)$ :

$$
\frac{\left\langle c_{0}, \sigma\right\rangle \stackrel{c_{0}}{\longrightarrow} \sigma^{\prime}\left\langle c_{2}, \sigma\right\rangle \stackrel{c_{2}}{\longrightarrow} \sigma^{\prime \prime}}{\left\langle\left(c_{0} ; c_{1}\right) \|\left(c_{2} ; c_{3}\right), \sigma\right\rangle \stackrel{\left\{c_{0}, c_{2}\right\}}{\longrightarrow}\left\langle c_{1} \| c_{3}, \sigma^{\prime} \uplus \sigma^{\prime \prime}\right\rangle}
$$

And we can get the following derivations of $\left(c_{0} \| c_{2}\right) ;\left(c_{1} \| c_{3}\right)$ :

$$
\frac{\left\langle c_{0}, \sigma\right\rangle \stackrel{c_{0}}{\longrightarrow} \sigma^{\prime}\left\langle c_{2}, \sigma\right\rangle \stackrel{c_{2}}{\longrightarrow} \sigma^{\prime \prime}}{\left\langle\left(c_{0} \| c_{2}\right) ;\left(c_{1} \| c_{3}\right), \sigma\right\rangle \stackrel{\left\{c_{0}, c_{2}\right\}}{\longrightarrow}\left\langle c_{1} \| c_{3}, \sigma^{\prime} \uplus \sigma^{\prime \prime}\right\rangle}
$$

So, it is obvious that $\left(c_{0} ; c_{1}\right) \|\left(c_{2} ; c_{3}\right) \sim\left(c_{0} \| c_{2}\right) ;\left(c_{1} \| c_{3}\right)$, for $c_{0}, c_{1}, c_{2}, c_{3} \in \mathbf{C o m}$, as desired.

Proposition 3.26. $c \|$ skip $\sim c$, for $c \in$ Com.

Proof. By use of the transition rules of skip and $\|$, we can get the following derivations of $c \|$ skip:

$$
\frac{\langle c, \sigma\rangle \stackrel{c}{\rightarrow} \sigma^{\prime} \quad\langle\text { skip, } \sigma\rangle \rightarrow \sigma}{c \| \text { skip, } \sigma\rangle \stackrel{c}{\rightarrow} \sigma^{\prime} \uplus \sigma}
$$

And it is obvious that:

$$
\frac{\langle c, \sigma\rangle \stackrel{c}{\rightarrow} \sigma^{\prime}}{c, \sigma\rangle \stackrel{c}{\rightarrow} \sigma^{\prime}}
$$

For $\sigma^{\prime} \uplus \sigma=\sigma^{\prime}$, it is obvious that $c \|$ skip $\sim c$, for $c \in \mathbf{C o m}$, as desired.

Lemma 3.27. For $c_{0}, c_{1} \in \mathbf{C o m}$,

1. $c_{0}\left\|c_{1} \sim c_{0}\right\|\left(\right.$ skip; $\left.c_{1}\right) \sim c_{0} ; c_{1}$

2. $c_{0}\left\|c_{1} \sim\left(\operatorname{skip} ; c_{0}\right)\right\| c_{1} \sim c_{1} ; c_{0}$. 
Proof. It is obvious by Proposition 3.25 and 3.26 .

From Lemma 3.27, we can see that the execution orders of $c_{0} \| c_{1}$ cause non-determinism, they can be executed in any sequential order or in parallel simultaneously. But, with the assistance of guards, the final states after the execution of $c_{0} \| c_{1}$ can be deterministic.

Proposition 3.28. For $c, c_{0}, c_{1} \in \mathbf{C o m}$,

1. $\alpha ! n \| \alpha ? n \sim \gamma_{\alpha}(n)$;

2. $(c ; \alpha ! n) \| \alpha ? n \sim c ; \gamma_{\alpha}(n)$;

3. $(c ; \alpha ? n) \| \alpha ! n \sim c ; \gamma_{\alpha}(n)$;

4. $\left(c_{0} ; \alpha ! n\right)\left\|\left(c_{1} ; \alpha ? n\right) \sim c_{0}\right\| c_{1} ; \gamma_{\alpha}(n)$;

5. $\left(c_{0} ; \alpha ? n\right)\left\|\left(c_{1} ; \alpha ! n\right) \sim c_{0}\right\| c_{1} ; \gamma_{\alpha}(n)$.

Proof. By use of the transition rules of $\|$, we can prove the above equations.

From Proposition 3.28, we can see that communications among parallel branches are eliminated and the parallelism is structured.

\subsection{Conflictions}

Corresponding to Figure 2, the program is:

$(1 ;$ (if $(\mathrm{b})$ then $(2 ; 3))) \|(4 ;$ (if $(\neg \mathrm{b})$ then $(5 ; 6)))$

Corresponding to Figure 8 , The program is:

if (b) then $(1 ; 2 ; 3) \| 4$ else $1 \|(4 ; 5 ; 6)$

We can prove that the above two programs are equivalent, and the confliction between parallel branches is eliminated and the parallelism is structured.

\subsection{Structuring Algorithm}

By PPL, We know that the truly concurrent graph can be structured. As an implementationindependent language, the structuring algorithm of PPL can be designed as follows:

1. Input the unstructured truly concurrent graph;

2. By use of PPL, implement the graph as a program;

3. By use of the laws of PPL, structure the program. 


\section{References}

[1] M. Danelutto, M. Torquati, and P. Kilpatrick. (2015). A green perspective on structured parallel programming. Euromicro International Conference on Parallel, Distributed and Network-Based Processing IEEE, 2015:430-437.

[2] M. Mccool, J. Reinders, and A. Robison. (2012). Structured parallel programming: patterns for efficient computation. Structured Parallel Programming. 2012:614-627.

[3] G. Winskel and M. Nielsen. (1995). Models for concurrency. In Samson Abramsky, Dov M. Gabbay,and Thomas S. E. Maibaum, Eds., Handbook of logic in Computer Science, 1995, vol. 4, Clarendon Press, Oxford, UK.

[4] M. Nielsen, G. D. Plotkin, and G. Winskel. (1981). Petri nets, event structures and domains, Part I. Theoret. Comput. Sci. 1981, 13, 85-108.

[5] G. Winskel. (1985). Event structures. In Petri Nets: Applications and Relationships to Other Models of Concurrency, Wilfried Brauer, Wolfgang Reisig, and Grzegorz Rozenberg, Eds., Lecture Notes in Computer Science, 1987, vol. 255, Springer, Berlin, 325-392.

[6] T. Elrad, and N. Francez. (1982). Decomposition of distributed programs into communication-closed layers. Science of Computer Programming 2.3(1982):155-173.

[7] W. Yuan, and Y. Sun. (1997). "SEQ OF PAR" structured parallel programming. Chinese J. Computers. 20(1997):230-237.

[8] Y. Wang. (2016). Algebraic laws for true concurrency. Manuscript, arXiv: 1611.09035.

[9] E. W. Dijkstra. (1968). Go to statement considered harmful. Communications of the ACM, 11(3), 147-148.

[10] O. J. Dahl, E. W. Dijkstra, C. A. R. Hoare. (1972). Structured programming. Programming and Computer Software, 18(7), 179-185.

[11] M. Cole. (2004). Why structured parallel programming matters. European Conference on Parallel Processing. Springer, Berlin, Heidelberg.

[12] L. V. Kale, N. Chrisochoides, J. Kohl, K. Yelick. (1995). Concurrency-based approaches to parallel programming. Office of scientific and technical information technical reports.

[13] R. D. Cosmo, Z. Li, V. Martin. (2003). Parallel programming with the ocamlp3l system, with applications to coupling numerical codes.

[14] J. Darlington, Y. Guo, H. W. To, J. Yang. (1995). Parallel skeletons for structured composition. ACM SIGPLAN Notices, 30(8), 19-28.

[15] M. McCool, A. D. Robison, J. Reinders. (2012). Structured parallel programming: patterns for efficient computation, Elsevier.

[16] J. Wiedermann. (1984). Parallel Turing machines. TR: RUU-CS-84-11.

[17] S. A. Cook. (1980). Towards a complexity theory of synchronous parallel computation. L'enseignement Math6matique XXVII, 27(2), 75-100. 
[18] R. E. Prather. (1977). Structured Turing machines. Information and Control.

[19] G. D. Plotkin. (2004). A structural approach to operational semantics. Journal of Logic and Algebraic Programming, 2004(60-61): 17-139.

[20] P. D. Mosses. (1991). Denotational semantics. Handbook of theoretical computer science, 1991(vol. B). MIT Press.

[21] G. Winskel. (1993). The formal semantics of programming languages. 1993. MIT Press. 